Research Article

\title{
Determinantal Representations of the Core Inverse and Its Generalizations with Applications
}

\author{
Ivan I. Kyrchei \\ Pidstrygach Institute for Applied Problems of Mechanics and Mathematics, NAS of Ukraine, Lviv, Ukraine \\ Correspondence should be addressed to Ivan I. Kyrchei; st260664@gmail.com
}

Received 11 June 2019; Accepted 17 August 2019; Published 1 October 2019

Academic Editor: Mehdi Ghatee

Copyright (c) 2019 Ivan I. Kyrchei. This is an open access article distributed under the Creative Commons Attribution License, which permits unrestricted use, distribution, and reproduction in any medium, provided the original work is properly cited.

In this paper, we give the direct method to find of the core inverse and its generalizations that is based on their determinantal representations. New determinantal representations of the right and left core inverses, the right and left core-EP inverses, and the DMP, MPD, and CMP inverses are derived by using determinantal representations of the Moore-Penrose and Drazin inverses previously obtained by the author. Since the Bott-Duffin inverse has close relation with the core inverse, we give its determinantal representation and its application in finding solutions of the constrained linear equations that is an analog of Cramer's rule. A numerical example to illustrate the main result is given.

\section{Introduction}

In the whole article, the notations $\mathbb{R}$ and $\mathbb{C}$ are reserved for fields of the real and complex numbers, respectively. $\mathbb{C}^{m \times n}$ stands for the set of all $m \times n$ matrices over $\mathbb{C}$. $\mathbb{C}_{r}^{m \times n}$ determines its subset of matrices with $\operatorname{rank} r$. For $\mathbf{A} \in \mathbb{C}^{m \times n}$, the symbols $\mathbf{A}^{T}, \mathbf{A}^{*}$, and $\operatorname{rk}(\mathbf{A})$ specify the transpose, the conjugate transpose, and the rank of $\mathbf{A}$, respectively. $|\mathbf{A}|$ or $\operatorname{det} \mathbf{A}$ stands for its determinant. A matrix $\mathbf{A} \in \mathbb{C}^{n \times n}$ is Hermitian if $\mathbf{A}^{*}=\mathbf{A}$.

$\mathbf{A}^{\dagger}$ means the Moore-Penrose inverse of $\mathbf{A} \in \mathbb{C}^{n \times m}$, i.e., the exclusive matrix $\mathbf{X}$ satisfying the following four equations:

$$
\begin{aligned}
\mathbf{A X A} & =\mathbf{A}, \\
\mathbf{X A X} & =\mathbf{X}, \\
(\mathbf{A X})^{*} & =\mathbf{A X}, \\
(\mathbf{X A})^{*} & =\mathbf{X A} .
\end{aligned}
$$

For $\mathbf{A} \in \mathbb{C}^{n \times n}$ with index Ind $\mathbf{A}=k$, i.e., the smallest positive number such that $\operatorname{rk}\left(\mathbf{A}^{k+1}\right)=\operatorname{rk}\left(\mathbf{A}^{k}\right)$, the Drazin inverse of $\mathbf{A}$, denoted by $\mathbf{A}^{d}$, is called the unique matrix $\mathbf{X}$ that satisfies equation (2) and the following equations:

$$
\begin{aligned}
\mathbf{A X} & =\mathbf{X A}, \\
\mathbf{X A}^{k+1} & =\mathbf{A}^{k}, \\
\mathbf{A}^{k+1} \mathbf{X} & =\mathbf{A}^{k} .
\end{aligned}
$$

In particular, if Ind $\mathbf{A}=1$, then the matrix $\mathbf{X}$ is called the group inverse and it is denoted by $\mathbf{X}=\mathbf{A}^{\#}$. If $\operatorname{Ind} \mathbf{A}=0$, then $\mathbf{A}$ is nonsingular and $\mathbf{A}^{d}=\mathbf{A}^{\dagger}=\mathbf{A}^{-1}$.

It is evident that if the condition (5) is fulfilled, then (6a) and (6b) are equivalent. We put both these conditions because they will be used below independently of each other and without the obligatory fulfillment of (5).

A matrix A satisfying the conditions $(i),(j), \ldots$ is called an $\{i, j, \ldots\}$-inverse of $\mathbf{A}$ and is denoted by $\mathbf{A}^{(i, j, \ldots)}$. The set of matrices $\mathbf{A}^{(i, j, \ldots)}$ is denoted by $\mathbf{A}\{i, j, \ldots\}$. In particular, $\mathbf{A}^{(1)}$ is called the inner inverse of $\mathbf{A}, \mathbf{A}^{(2)}$ is called the outer inverse of $\mathbf{A}, \mathbf{A}^{(1,2)}$ is called the reflexive inverse of $\mathbf{A}$, $\mathbf{A}^{(1,2,3,4)}$ is its Moore-Penrose inverse, etc.

For an arbitrary matrix $\mathbf{A} \in \mathbb{C}^{m \times n}$, we denote by

(i) $\mathcal{N}(\mathbf{A})=\left\{\mathbf{x} \in \mathbb{C}^{n \times 1}: \mathbf{A x}=0\right\}$, the kernel (or the null space) of $\mathbf{A}$ 
(ii) $\mathscr{C}(\mathbf{A})=\left\{\mathbf{y} \in \mathbb{C}^{m \times 1}: \mathbf{y}=\mathbf{A x}, \mathbf{x} \in \mathbb{C}^{n \times 1}\right\}$, the column space (or the range space) of $\mathbf{A}$

(iii) $\mathscr{R}(\mathbf{A})=\left\{\mathbf{y} \in \mathbb{C}^{1 \times n}: \mathbf{y}=\mathbf{x A}, \mathbf{x} \in \mathbb{C}^{1 \times m}\right\}$, the row space of $\mathbf{A}$

$\mathbf{P}_{A}:=\mathbf{A} \mathbf{A}^{\dagger}$ and $\mathbf{Q}_{A}:=\mathbf{A}^{\dagger} \mathbf{A}$ are the orthogonal projectors onto the range of $\mathbf{A}$ and the range of $\mathbf{A}^{*}$, respectively.

The core inverse was introduced by Baksalary and Trenkler in [1]. Later, it was investigated by Xu et al. in [2], among others. Rakić et al. in [3] generalized the core inverse of a complex matrix to the case of an element in a ring.

Definition 1 [1]. A matrix $\mathbf{X} \in \mathbb{C}^{n \times n}$ is called the core inverse of $\mathbf{A} \in \mathbb{C}^{n \times n}$ if it satisfies the conditions

$$
\begin{aligned}
\mathbf{A X} & =\mathbf{P}_{A}, \\
\mathscr{C}(\mathbf{X}) & =\mathscr{C}(\mathbf{A}) .
\end{aligned}
$$

When such matrix $\mathbf{X}$ exists, it is denoted by $\mathbf{A}^{\oplus}$.

In 2014, the core inverse was extended to the core-EP inverse defined by Manjunatha Prasad and Mohana [4]. Other generalizations of the core inverse were recently introduced for $n \times n$ complex matrices, namely, BT inverses [5], DMP inverses [6], CMP inverses [7], etc. The characterizations, computing methods, some applications of the core inverse, and its generalizations were recently investigated in complex matrices and rings (see, e.g., [3, 8-18]).

In contrast to the inverse matrix that has a definitely determinantal representation in terms of cofactors, for generalized inverse matrices there exist different determinantal representations as a result of the search of their more applicable explicit expressions (see, e.g., [19-25]). In this paper, we get new determinantal representations of the core inverse and its generalizations by using the author's recently obtained determinantal representations of the Moore-Penrose inverse and the Drazin inverse over the quaternion skew field and the field of complex numbers as a special case $[26,27]$.

The results concerning quaternion matrices have been achieved thanks to the theory of row-column determinants introduced in $[28,29]$. Within the framework of the theory of row-column determinants, determinantal representations of various kind of generalized inverses, generalized inverse solutions (analogs of Cramer's rule) of quaternion matrix equations have been derived by the author (see, e.g., [30-38]) and by other researchers (see, e.g., [39-41]).

Note that a determinantal representation of the core-EP generalized inverse in complex matrices has been derived in [4] based on the determinantal representation of a reflexive inverse obtained in $[19,20]$.

One of possible applications of the determinantal representations of the inverse matrix is Cramer's rule to find solutions of systems of linear equations. Determinantal representations of generalized inverses have similar applications. In this paper, we derive the determinantal representation of the Bott-Duffin inverse that has close relation with the core inverse, and apply it to obtain Cramer's rule for the constrained linear equations.

The paper is organized as follows. In Section 2, we start with preliminary introduction of determinantal representations of the Moore-Penrose inverse and the Drazin inverse. In the next sections, we give determinantal representations of the core inverse and its generalizations. In particular, determinantal representations of the right and left core inverses are established in Section 3, of the right and left core-EP inverses in Section 4 , and of the DMP inverse and its dual MPD inverse in Section 5. Determinantal representations of the CMP inverse are obtained in Section 6. In Section 7, we derive Cramer's rule for the constrained linear equations by using the determinantal representation of the Bott-Duffin inverse that is same as for the right core inverse. A numerical example to illustrate the main results is considered in Section 8. Finally, in Section 9, the conclusions are drawn.

\section{Preliminaries}

Let $\alpha:=\left\{\alpha_{1}, \ldots, \alpha_{k}\right\} \subseteq\{1, \ldots, m\}$ and $\beta:=\left\{\beta_{1}, \ldots, \beta_{k}\right\} \subseteq$ $\{1, \ldots, n\}$ be subsets with $1 \leq k \leq \min \{m, n\}$. The submatrix of $\mathbf{A} \in \mathbb{C}^{m \times n}$ with rows and columns indexed by $\alpha$ and $\beta$, respectively, and denoted by $\mathbf{A}_{\beta}^{\alpha}$. Then, $\mathbf{A}_{\alpha}^{\alpha}$ is a principal submatrix of $\mathbf{A}$ with rows and columns indexed by $\alpha$, and $|\mathbf{A}|_{\alpha}^{\alpha}$ is the corresponding principal minor of the determinant $|\mathbf{A}|$. Suppose that

$$
L_{k, n}:=\left\{\alpha: \alpha=\left(\alpha_{1}, \ldots, \alpha_{k}\right), \quad 1 \leq \alpha_{1}<\ldots<\alpha_{k} \leq n\right\},
$$

stands for the collection of strictly increasing sequences of $1 \leq k \leq n$ integers chosen from $\{1, \ldots, n\}$. For fixed $i \in \alpha$ and $j \in \beta$, put $I_{r, m}\{i\}:=\left\{\alpha: \alpha \in L_{r, m}, i \in \alpha\right\}$ and $J_{r, n}\{j\}:=\{\beta: \beta$ $\left.\in L_{r, n}, j \in \beta\right\}$.

Denote by $\mathbf{a}_{. j}$ and $\mathbf{a}_{. j}^{*}, \mathbf{a}_{i .}$ and $\mathbf{a}_{i .}^{*}$ the $j$ th columns and the $i$ th rows of $\mathbf{A}$ and $\mathbf{A}^{*}$, respectively. By $\mathbf{A}_{i .}$ (b) and $\mathbf{A}_{. j}(\mathbf{c})$, we denote the matrices obtained from $\mathbf{A}$ by replacing its $i$ th row with the row $\mathbf{b}$ and its $j$ th column with the column c.

Theorem 1 [21]. If $\mathbf{A} \in \mathbb{C}_{r}^{m \times n}$, then the Moore-Penrose inverse $\mathbf{A}^{\dagger}=\left(a_{i j}^{\dagger}\right) \in \mathbb{C}^{n \times m}$ possess the determinantal representations

$$
\begin{aligned}
& a_{i j}^{\dagger}=\frac{\sum_{\beta \in J_{r, n}\{i\}}\left|\left(\mathbf{A}^{*} \mathbf{A}\right)_{. i}\left(\mathbf{a}_{. j}^{*}\right)\right|_{\beta}^{\beta}}{\sum_{\beta \in J_{r, n}}\left|\mathbf{A}^{*} \mathbf{A}\right|_{\beta}^{\beta}}, \\
& a_{i j}^{\dagger}=\frac{\sum_{\alpha \in I_{r, m}\{j\}}\left|\left(\mathbf{A} \mathbf{A}^{*}\right)_{j .}\left(\mathbf{a}_{i .}^{*}\right)\right|_{\alpha}^{\alpha}}{\sum_{\alpha \in I_{r, m}}\left|\mathbf{A} \mathbf{A}^{*}\right|_{\alpha}^{\alpha}} .
\end{aligned}
$$

Remark 1. For an arbitrary full-rank matrix $\mathbf{A} \in \mathbb{C}_{r}^{m \times n}$, a row-vector $\mathbf{b} \in \mathbb{H}^{1 \times m}$, and a column-vector $\mathbf{c} \in \mathbb{H}^{n \times 1}$, we mean, respectively, 


$$
\begin{aligned}
\left|\left(\mathbf{A A}^{*}\right)_{i .}(\mathbf{b})\right| & =\sum_{\alpha \in I_{m, m}\{i\}}\left|\left(\mathbf{A A}^{*}\right)_{i .}(\mathbf{b})\right|_{\alpha}^{\alpha}, \quad i=1, \ldots, m, \\
\left|\mathbf{A A}^{*}\right| & =\sum_{\alpha \in I_{m, m}}\left|\mathbf{A A}^{*}\right|_{\alpha}^{\alpha}, \quad \text { when } r=m, \\
\left|\left(\mathbf{A}^{*} \mathbf{A}\right)_{. j}(\mathbf{c})\right| & =\sum_{\beta \in J_{n, n}\{j\}}\left|\left(\mathbf{A}^{*} \mathbf{A}\right)_{. j}(\mathbf{c})\right|_{\beta}^{\beta}, \quad j=1, \ldots, n, \\
\left|\mathbf{A}^{*} \mathbf{A}\right| & =\sum_{\beta \in J_{n, n}}\left|\mathbf{A}^{*} \mathbf{A}\right|_{\beta}^{\beta}, \quad \text { when } r=n .
\end{aligned}
$$

Corollary 1 [21]. Let $\mathbf{A} \in \mathbb{C}_{r}^{m \times n}$. Then, the following determinantal representations can be obtained:

(i) For the projector $\mathbf{Q}_{A}=\left(q_{i j}\right)_{n \times n}$,

$$
q_{i j}=\frac{\sum_{\beta \in J_{r, n}\{i\}}\left|\left(\mathbf{A}^{*} \mathbf{A}\right)_{. i}\left(\dot{\mathbf{a}}_{. j}\right)\right|_{\beta}^{\beta}}{\sum_{\beta \in J_{r, n}}\left|\mathbf{A}^{*} \mathbf{A}\right|_{\beta}^{\beta}}=\frac{\sum_{\alpha \in I_{r, n}\{j\}}\left|\left(\mathbf{A}^{*} \mathbf{A}\right)_{j .}\left(\dot{\mathbf{a}}_{i .}\right)\right|_{\alpha}^{\alpha}}{\sum_{\alpha \in I_{r, n}}\left|\mathbf{A}^{*} \mathbf{A}\right|_{\alpha}^{\alpha}},
$$

where $\dot{\mathbf{a}}_{j}$ is the jth column and $\dot{\mathbf{a}}_{i .}$ is the ith row of $\mathbf{A}^{*} \mathbf{A}$.

(ii) For the projector $\mathbf{P}_{A}=\left(p_{i j}\right)_{m \times m}$,

$$
p_{i j}=\frac{\sum_{\alpha \in I_{r, m}\{j\}}\left|\left(\mathbf{A A}^{*}\right)_{j .}\left(\ddot{\mathbf{a}}_{i .}\right)\right|_{\alpha}^{\alpha}}{\sum_{\alpha \in I_{r, m}}\left|\mathbf{A} \mathbf{A}^{*}\right|_{\alpha}^{\alpha}}=\frac{\sum_{\beta \in J_{r, m}\{i\}}\left|\left(\mathbf{A A}^{*}\right)_{. i}\left(\ddot{\mathbf{a}}_{. j}\right)\right|_{\beta}^{\beta}}{\sum_{\beta \in J_{r, m}}\left|\mathbf{A} \mathbf{A}^{*}\right|_{\beta}^{\beta}},
$$

where $\ddot{\mathbf{a}}_{i .}$ is the ith row and $\ddot{\mathbf{a}}_{. j}$ is the jth column of $\mathbf{A A}^{*}$.

The following lemma gives determinantal representations of the Drazin inverse in complex matrices.

Lemma 1 [22]. Let $\mathbf{A} \in \mathbb{C}^{n \times n}$ with $\operatorname{Ind} \mathbf{A}=k$ and $r k \mathbf{A}^{k+1}=$ $r k \mathbf{A}^{k}=r$. Then, the determinantal representations of the Drazin inverse $\mathbf{A}^{d}=\left(a_{i j}^{d}\right) \in \mathbb{C}^{n \times n}$ are

$$
\begin{aligned}
a_{i j}^{d} & =\frac{\sum_{\beta \in J_{r, n}\{i\}}\left|\left(\mathbf{A}^{k+1}\right)_{. i}\left(\mathbf{a}_{. j}^{(k)}\right)\right|_{\beta}^{\beta}}{\sum_{\beta \in J_{r, n}}\left|\mathbf{A}^{k+1}\right|_{\beta}^{\beta}} \\
& =\frac{\sum_{\alpha \in I_{r, n}\{j\}}\left|\left(\mathbf{A}^{k+1}\right)_{j .}\left(\mathbf{a}_{i .}^{(k)}\right)\right|_{\alpha}^{\alpha}}{\sum_{\alpha \in I_{r, n}}\left|\mathbf{A}^{k+1}\right|_{\alpha}^{\alpha}},
\end{aligned}
$$

where $\mathbf{a}_{i .}^{(k)}$ is the ith row and $\mathbf{a}_{. j}^{(k)}$ is the jth column of $\mathbf{A}^{k}$.
Corollary 2 [22]. Let $\mathbf{A} \in \mathbb{C}^{n \times n}$ with Ind $\mathbf{A}=1$ and $r k \mathbf{A}^{2}=$ $r k \mathbf{A}=r$. Then, the determinantal representations of the group inverse $\mathbf{A}^{\#}=\left(a_{i j}^{\#}\right) \in \mathbb{C}^{n \times n}$ are

$$
a_{i j}^{\#}=\frac{\sum_{\beta \in J_{r, n}\{i\}}\left|\left(\mathbf{A}^{2}\right)_{. i}\left(\mathbf{a}_{. j}\right)\right|_{\beta}^{\beta}}{\sum_{\beta \in J_{r, n}}\left|\mathbf{A}^{2}\right|_{\beta}^{\beta}}=\frac{\sum_{\alpha \in I_{r, n}\{j\}}\left(\mathbf{A}^{2}\right)_{j .}\left(\mathbf{a}_{i .}\right)_{\alpha}^{\alpha}}{\sum_{\alpha \in I_{r, n}}\left|\mathbf{A}^{2}\right|_{\alpha}^{\alpha}} .
$$

\section{Determinantal Representations of the Core Inverses}

Together with the core inverse in [3], it was introduced the dual core inverse. Since the both these core inverses are equipollent and they are different only in the position relative to the inducting matrix $\mathbf{A}$, we propose to call them as the right and left core inverses regarding to their positions. So, according to [1], we have the following definition that is equivalent to Definition 1.

Definition 2. A matrix $\mathbf{X} \in \mathbb{C}^{n \times n}$ is said to be the right core inverse matrix of $\mathbf{A} \in \mathbb{C}^{n \times n}$ if it satisfies the conditions

$$
\begin{aligned}
\mathbf{A X} & =\mathbf{P}_{A}, \\
\mathscr{C}(\mathbf{X}) & =\mathscr{C}(\mathbf{A}) .
\end{aligned}
$$

When such matrix $\mathbf{X}$ exists, it is denoted by $\mathbf{A}^{\oplus}$.

The following definition of the left core inverse can be given that is equivalent to the introduced dual core inverse [3].

Definition 3. A matrix $\mathbf{X} \in \mathbb{C}^{n \times n}$ is said to be the left core inverse matrix of $\mathbf{A} \in \mathbb{C}^{n \times n}$ if it satisfies the conditions

$$
\begin{aligned}
\mathbf{X A} & =\mathbf{Q}_{A}, \\
\mathscr{R}(\mathbf{X}) & =\mathscr{R}(\mathbf{A}) .
\end{aligned}
$$

When such matrix $\mathbf{X}$ exists, it is denoted by $\mathbf{A}_{\oplus}$.

Remark 2. In [42], the conditions of the dual core inverse are given as follows:

$$
\begin{gathered}
\mathbf{A}_{\oplus} \mathbf{A}=\mathbf{P}_{A^{*}}, \\
\mathscr{C}\left(\mathbf{A}_{\oplus}\right) \subseteq \mathscr{C}\left(\mathbf{A}^{*}\right) .
\end{gathered}
$$

Since $\mathbf{P}_{A^{*}}=\mathbf{A}^{*}\left(\mathbf{A}^{*}\right)^{\dagger}=\left(\mathbf{A}^{\dagger} \mathbf{A}\right)^{*}=\mathbf{A}^{\dagger} \mathbf{A}=\mathbf{Q}_{A}$ and $\mathscr{R}(\mathbf{A})$ $=\mathscr{C}\left(\mathbf{A}^{*}\right)$, then these conditions and (17) are analogous.

Remark 3. In Definitions 2 and 3, we purposely state that these definitions concern with matrices, since the right and core inverses in a ring were introduced recently in [43]. The notions of the right and left core inverse matrices, introduced here, and of the right and left core inverses in a ring, introduced in [43], have no direct connection with each other.

According to [1], we introduce the following sets of matrices: 


$$
\begin{aligned}
& \mathbb{C}_{n}^{\mathrm{CM}}=\left\{\mathbf{A} \in \mathbb{C}^{n \times n}: r k \mathbf{A}^{2}=\mathrm{rk} \mathbf{A}\right\} \\
& \mathbb{C}_{n}^{\mathrm{EP}}=\left\{\mathbf{A} \in \mathbb{C}^{n \times n}: \mathbf{A}^{\dagger} \mathbf{A}=\mathbf{A} \mathbf{A}^{\dagger}\right\}=\left\{\mathbf{A} \in \mathbb{C}^{n \times n}: \mathscr{C}(\mathbf{A})=\mathscr{C}\left(\mathbf{A}^{*}\right)\right\}
\end{aligned}
$$

The matrices from $\mathbb{C}_{n}^{\mathrm{CM}}$ are called group matrices or core matrices. If $\mathbf{A} \in \mathbb{C}_{n}^{\mathrm{EP}}$, then clearly $\mathbf{A}^{\dagger}=\mathbf{A}^{\#}$. It is known that the core inverses of $\mathbf{A} \in \mathbb{C}^{n \times n}$ exist if and only if $\mathbf{A} \in \mathbb{C}_{n}^{\mathrm{CM}}$ or Ind $\mathbf{A}=1$. Moreover, if $\mathbf{A}$ is nonsingular, Ind $\mathbf{A}=0$, then its core inverses are the usual inverse. According to [1], we have the following representations of the right and left core inverses.

Lemma 2 [1]. Let $\mathbf{A} \in \mathbb{C}_{n}^{C M}$. Then,

$$
\begin{aligned}
& \mathbf{A}^{\oplus}=\mathbf{A}^{\#} \mathbf{A} \mathbf{A}^{\dagger}, \\
& \mathbf{A}_{\oplus}=\mathbf{A}^{\dagger} \mathbf{A} \mathbf{A}^{\#} .
\end{aligned}
$$

Remark 4. In Theorems 2 and 3, we will suppose that $\mathbf{A} \in \mathbb{C}_{n}^{\mathrm{CM}}$ but $\mathbf{A} \notin \mathbb{C}_{n}^{\mathrm{EP}}$. if $\mathbf{A} \in \mathbb{C}_{n}^{\mathrm{CM}}$ and $\mathbf{A} \in \mathbb{C}_{n}^{\mathrm{EP}}$ (in particular, $\mathbf{A}$ is Hermitian), then from Lemma 2 and the definitions of the Moore-Penrose and group inverses it follows that $\mathbf{A}^{\boxplus}=\mathbf{A}_{\oplus}=\mathbf{A}^{\#}=\mathbf{A}^{\dagger}$.

Theorem 2. Let $\mathbf{A} \in \mathbb{C}_{n}^{C M}$ and $r k \mathbf{A}^{2}=r k \mathbf{A}=s$. Then, its right core inverse matrix $\mathbf{A}^{\boxplus}=\left(a_{i j}^{\circledast, r}\right)$ has the following determinantal representations:

$$
\begin{aligned}
a_{i j}^{\oplus, r} & =\frac{\sum_{\alpha \in I_{s, n}\{j\}}\left|\left(\mathbf{A} \mathbf{A}^{*}\right)_{j .}\left(\mathbf{u}_{i .}^{(1)}\right)\right|_{\alpha}^{\alpha}}{\sum_{\beta \in J_{s, n}}\left|\mathbf{A}^{2}\right|_{\beta}^{\beta} \sum_{\alpha \in I_{s, n}}\left|\mathbf{A} \mathbf{A}^{*}\right|_{\alpha}^{\alpha}} \\
& =\frac{\sum_{\beta \in J_{s, n}\{i\}}\left|\left(\mathbf{A}^{2}\right)_{. i}\left(\mathbf{u}_{. j}^{(2)}\right)\right|_{\beta}^{\beta}}{\sum_{\beta \in J_{s, n}}\left|\mathbf{A}^{2}\right|_{\beta}^{\beta} \sum_{\alpha \in I_{s, n}}\left|\mathbf{A} \mathbf{A}^{*}\right|_{\alpha}^{\alpha}}
\end{aligned}
$$

where

$$
\begin{aligned}
& \mathbf{u}_{i .}^{(1)}=\left[\sum_{\beta \in J_{s, n}\{i\}}\left|\left(\mathbf{A}^{2}\right)_{. i}\left(\widetilde{\mathbf{a}}_{. f}\right)\right|_{\beta}^{\beta}\right] \in \mathbb{C}^{1 \times n}, \quad f=1, \ldots, n, \\
& \mathbf{u}_{. j}^{(2)}=\left[\sum_{\alpha \in I_{s, n}\{j\}}\left|\left(\mathbf{A A}^{*}\right)_{j .}\left(\widetilde{\mathbf{a}}_{l .}\right)\right|_{\alpha}^{\alpha}\right] \in \mathbb{C}^{n \times 1}, \quad l=1, \ldots, n,
\end{aligned}
$$

are the row and column vectors, respectively. Here, $\widetilde{\mathbf{a}}_{. f}$ and $\widetilde{\mathbf{a}}_{l}$ are the fth column and lth row of $\widetilde{A}:=\mathbf{A}^{2} \mathbf{A}^{*}$.

Proof. Taking into account (20), we have for $\mathbf{A}^{\oplus}$

$$
a_{i j}^{\oplus, r}=\sum_{l=1}^{n} \sum_{f=1}^{n} a_{i l}^{\#} a_{l f} a_{f j}^{\dagger}
$$

By substituting (15) and (12) in (25), we obtain

$$
\begin{aligned}
a_{i j}^{\oplus, r} & =\sum_{l=1}^{n} \frac{\sum_{f=1}^{n} \sum_{\beta \in J_{s, n}\{i\}}\left|\left(\mathbf{A}^{2}\right)_{. i}\left(\mathbf{a}_{. f}\right)\right|_{\beta}^{\beta} a_{f l}}{\sum_{\beta \in J_{s, n}}\left|\mathbf{A}^{2}\right|_{\beta}^{\beta}} \frac{\sum_{\alpha \in I_{s, n}\{j\}}\left|\left(\mathbf{A A}^{*}\right)_{j .}\left(\mathbf{a}_{l .}^{*}\right)\right|_{\alpha}^{\alpha}}{\sum_{\alpha \in I_{s, n}}\left|\mathbf{A} \mathbf{A}^{*}\right|_{\alpha}^{\alpha}} \\
& =\frac{\sum_{f=1}^{n} \sum_{l=1}^{n} \sum_{\beta \in J_{s, n}\{j\}}\left|\left(\mathbf{A}^{2}\right)_{. j}\left(\mathbf{e}_{. f}\right)\right|_{\beta}^{\beta} \widetilde{a}_{f l} \sum_{\alpha \in I_{s, n}\{j\}}\left|\left(\mathbf{A A}^{*}\right)_{j .}\left(\mathbf{e}_{l .}\right)\right|_{\alpha}^{\alpha}}{\sum_{\beta \in J_{s, n}}\left|\mathbf{A}^{2}\right|_{\beta}^{\beta} \sum_{\alpha \in I_{s, n}}\left|\mathbf{A} \mathbf{A}^{*}\right|_{\alpha}^{\alpha}},
\end{aligned}
$$

where $\mathbf{e}_{. l}$ and $\mathbf{e}_{l}$ are the unit column and row vectors, respectively, such that all their components are 0 , except the $l$ th components which are $1 ; \widetilde{a}_{l f}$ is the $(l f)$ th element of the matrix $\widetilde{\mathbf{A}}:=\mathbf{A}^{2} \mathbf{A}^{*}$.

Let

$$
\begin{array}{r}
u_{i l}^{(1)}:=\sum_{f=1}^{n} \sum_{\beta \in J_{s, n}\{i\}}\left|\left(\mathbf{A}^{2}\right)_{. i}\left(\mathbf{e}_{. f}\right)\right|_{\beta}^{\beta} \widetilde{a}_{f l}=\sum_{\beta \in J_{s, n}\{i\}}\left|\left(\mathbf{A}^{2}\right)_{. i}\left(\tilde{\mathbf{A}}_{l l}\right)\right|_{\beta}^{\beta}, \\
i, l=1, \ldots, n .
\end{array}
$$

Construct the matrix $\mathbf{U}_{1}=\left(u_{i l}^{(1)}\right) \in \mathbb{H}^{n \times n}$. It follows that

$$
\sum_{l} u_{i l}^{(1)} \sum_{\alpha \in I_{s, n}\{j\}}\left|\left(\mathbf{A A}^{*}\right)_{j .}\left(\mathbf{e}_{l .}\right)\right|_{\alpha}^{\alpha}=\sum_{\alpha \in I_{s, n}\{j\}}\left|\left(\mathbf{A A}^{*}\right)_{j .}\left(\mathbf{u}_{i .}^{(1)}\right)\right|_{\alpha}^{\alpha}
$$

where $\mathbf{u}_{i .}^{(1)}$ is the $i$ th row of $\mathbf{U}_{1}$. So, we get (22).

If we first consider

$$
\begin{array}{r}
u_{i f}^{(2)}:=\sum_{l} \tilde{a}_{f l} \sum_{\alpha \in I_{s, n}\{j\}}\left|\left(\mathbf{A A}^{*}\right)_{j .}\left(\mathbf{e}_{l .}\right)\right|_{\alpha}^{\alpha}=\sum_{\alpha \in I_{s, n}\{j\}}\left|\left(\mathbf{A A}^{*}\right)_{j .}\left(\tilde{a}_{f .}\right)\right|_{\alpha}^{\alpha}, \\
f, j=1, \ldots, n,
\end{array}
$$

and construct the matrix $\mathbf{U}_{2}=\left(u_{i f}^{(2)}\right) \in \mathbb{T}^{n \times n}$, then from

$$
\sum_{f=1}^{n} \sum_{\beta \in J_{s, n}\{i\}}\left|\left(\mathbf{A}^{2}\right)_{. i}\left(\mathbf{e}_{. f}\right)\right|_{\beta}^{\beta} u_{i f}^{(2)}=\sum_{\beta \in J_{s, n}\{i\}}\left|\left(\mathbf{A}^{2}\right)_{. i}\left(\mathbf{u}_{. f}^{(2)}\right)\right|_{\beta}^{\beta} \text {, }
$$

it follows (22).

Taking into account (21), the following theorem on the determinantal representation of the left core inverse can be proved similarly.

Theorem 3. Let $\mathbf{A} \in \mathbb{C}_{n}^{C M}$ and $r k \mathbf{A}^{2}=r k \mathbf{A}=s$. Then, for its left core inverse matrix $\left(\mathbf{A}_{\oplus}\right)=\left(a_{i j}^{\oplus l}\right)$, we have

$$
a_{i j}^{\oplus, l}=\frac{\sum_{\alpha \in I_{s, n}\{j\}}\left|\left(\mathbf{A}^{2}\right)_{j .}\left(\mathbf{v}_{i .}^{(1)}\right)\right|_{\alpha}^{\alpha}}{\sum_{\beta \in J_{s, n}}\left|\mathbf{A}^{*} \mathbf{A}\right|_{\beta}^{\beta} \sum_{\alpha \in I_{s, n}}\left|\mathbf{A}^{2}\right|_{\alpha}^{\alpha}}=\frac{\sum_{\beta \in J_{s, n}\{i\}}\left|\left(\mathbf{A}^{*} \mathbf{A}\right)_{. i}\left(\mathbf{v}_{. j}^{(2)}\right)\right|_{\beta}^{\beta}}{\sum_{\beta \in J_{s, n}}\left|\mathbf{A}^{*} \mathbf{A}\right|_{\beta}^{\beta} \sum_{\alpha \in I_{s, n}}\left|\mathbf{A}^{2}\right|_{\alpha}^{\alpha},}
$$


where

$$
\begin{aligned}
& \mathbf{v}_{i .}^{(1)}=\left[\sum_{\beta \in J_{s, n}\{i\}}\left|\left(\mathbf{A}^{*} \mathbf{A}\right)_{. i}\left(\bar{a}_{. f}\right)\right|_{\beta}^{\beta}\right] \in \mathbb{H}^{1 \times n}, \quad f=1, \ldots, n, \\
& \mathbf{v}_{. j}^{(2)}=\left[\sum_{\alpha \in I_{s, n}\{j\}}\left|\left(\mathbf{A}^{2}\right)_{j .}\left(\bar{a}_{l .}\right)\right|_{\alpha}^{\alpha}\right] \in \mathbb{H}^{n \times 1}, \quad l=1, \ldots, n .
\end{aligned}
$$

Here, $\overline{\mathbf{a}}_{f}$ and $\overline{\mathbf{a}}_{l}$. are the fth column and lth row of $\overline{\mathbf{A}}:=\mathbf{A}^{*} \mathbf{A}^{2}$.

\section{Determinantal Representations of the Core- EP Inverses}

Similar to [4], we introduce two core-EP inverses.

Definition 4. A matrix $\mathbf{X} \in \mathbb{C}^{n \times n}$ is said to be the right coreEP inverse of $\mathbf{A} \in \mathbb{C}^{n \times n}$ if it satisfies the conditions

$$
\begin{aligned}
& \mathbf{X A X}=\mathbf{A}, \\
& \mathscr{C}(\mathbf{X})=\mathscr{C}\left(\mathbf{X}^{*}\right)=\mathscr{C}\left(\mathbf{A}^{d}\right) .
\end{aligned}
$$

It is denoted by $\mathbf{A}^{\oplus}$.

Definition 5. A matrix $\mathbf{X} \in \mathbb{C}^{n \times n}$ is said to be the left core-EP inverse of $\mathbf{A} \in \mathbb{C}^{n \times n}$ if it satisfies the conditions

$$
\begin{aligned}
& \mathbf{X A X}=\mathbf{A}, \\
& \mathscr{R}(\mathbf{X})=\mathscr{R}\left(\mathbf{X}^{*}\right)=\mathscr{R}\left(\mathbf{A}^{d}\right) .
\end{aligned}
$$

It is denoted by $\mathbf{A}_{\oplus}$.

Remark 5. Since $\mathscr{C}\left(\left(\mathbf{A}^{*}\right)^{d}\right)=\mathscr{R}\left(\mathbf{A}^{d}\right)$, then the left core-EP inverse $\mathbf{A}_{\oplus}$ of $\mathbf{A} \in \mathbb{C}^{n \times n}$ is similar to the $*$ core inverse introduced in [4], and the dual core-EP inverse introduced in [42].

According to [4], we have the following representations the core-EP inverses of $\mathbf{A} \in \mathbb{C}^{n \times n}$ :

$$
\begin{aligned}
& \mathbf{A}^{\oplus}=\mathbf{A}^{\{2,3,6 a\}}, \mathscr{C}\left(\mathbf{A}^{\oplus}\right) \subseteq \mathscr{C}\left(\mathbf{A}^{k}\right), \\
& \mathbf{A}_{\oplus}=\mathbf{A}^{\{2,4,6 b\}}, \mathscr{R}\left(\mathbf{A}_{\oplus}\right) \subseteq \mathscr{R}\left(\mathbf{A}^{k}\right) .
\end{aligned}
$$

Thanks to [42], the following representations of the coreEP inverses will be used for their determinantal representations.

Lemma 3. Let $\mathbf{A} \in \mathbb{C}^{n \times n}$ and Ind $\mathbf{A}=k$. Then,

$$
\begin{aligned}
& \mathbf{A}^{\oplus}=\mathbf{A}^{k}\left(\mathbf{A}^{k+1}\right)^{\dagger}, \\
& \mathbf{A}_{\oplus}=\left(\mathbf{A}^{k+1}\right)^{\dagger} \mathbf{A}^{k} .
\end{aligned}
$$

Moreover, if Ind $\mathbf{A}=1$, then we have the following representations of the right and left core inverse matrices:

$$
\begin{aligned}
& \mathbf{A}^{\oplus}=\mathbf{A}\left(\mathbf{A}^{2}\right)^{\dagger}, \\
& \mathbf{A}_{\oplus}=\left(\mathbf{A}^{2}\right)^{\dagger} \mathbf{A} .
\end{aligned}
$$

Theorem 4. Suppose $\mathbf{A} \in \mathbb{C}^{n \times n}$, Ind $\mathbf{A}=k$, and $r k \mathbf{A}^{k}=s$, and

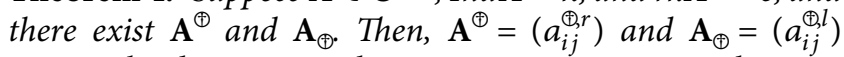
possess the determinantal representations, respectively,

$$
\begin{aligned}
& a_{i j}^{\oplus, r}=\frac{\sum_{\alpha \in I_{s, n}\{j\}}\left|\left(\mathbf{A}^{k+1}\left(\mathbf{A}^{k+1}\right)^{*}\right)_{j .}\left(\widehat{\mathbf{a}}_{i .}\right)\right|_{\alpha}^{\alpha}}{\sum_{\alpha \in I_{s, n}}\left|\mathbf{A}^{k+1}\left(\mathbf{A}^{k+1}\right)^{*}\right|_{\alpha}^{\alpha}}, \\
& a_{i j}^{\oplus, l}=\frac{\sum_{\beta \in J_{s, n}\{i\}}\left|\left(\left(\mathbf{A}^{k+1}\right)^{*} \mathbf{A}^{k+1}\right)_{. i}\left(\check{\mathbf{a}}_{. j}\right)\right|_{\beta}^{\beta}}{\sum_{\beta \in J_{s, n}}\left|\left(\mathbf{A}^{k+1}\right)^{*} \mathbf{A}^{k+1}\right|_{\beta}^{\beta}},
\end{aligned}
$$

where $\widehat{a}_{i}$ is the ith row of $\widehat{\mathbf{A}}=\mathbf{A}^{k}\left(\mathbf{A}^{k+1}\right)^{*}$ and $\check{\mathbf{a}}_{. j}$ is the jth column of $\check{\mathbf{A}}=\left(\mathbf{A}^{k+1}\right)^{*} \mathbf{A}^{k}$.

Proof. Consider $\left(\mathbf{A}^{k+1}\right)^{\dagger}=\left(a_{i j}^{(k+1, \dagger)}\right)$ and $\mathbf{A}^{k}=\left(a_{i j}^{(k)}\right)$. By (36),

$$
a_{i j}^{\oplus, r}=\sum_{t=1}^{n} a_{i t}^{(k)} a_{t j}^{(k+1, \dagger)}
$$

Taking into account (10) for the determinantal representation of $\left(\mathbf{A}^{k+1}\right)^{\dagger}$, we get

$$
a_{i j}^{\oplus, r}=\sum_{t=1}^{n} a_{i t}^{(k)} \frac{\sum_{\alpha \in I_{s, n}\{j\}}\left|\left(\mathbf{A}^{k+1}\left(\mathbf{A}^{k+1}\right)^{*}\right)_{j .}\left(\mathbf{a}_{t .}^{(k+1, \dagger)}\right)\right|_{\alpha}^{\alpha}}{\sum_{\alpha \in I_{r, m}}\left|\mathbf{A}^{k+1}\left(\mathbf{A}^{k+1}\right)^{*}\right|_{\alpha}^{\alpha}},
$$

where $\mathbf{a}_{t .}^{(k+1)}$ is the $t$ th row of $\left(\mathbf{A}^{k+1}\right)^{*}$. Since $\sum_{t=1}^{n} a_{i t}^{(k)} \mathbf{a}_{t .}^{(k+1)}=$ $\widehat{\mathbf{a}}_{i}$, then it follows (40).

The determinantal representation (41) can be obtained similarly by integrating (9) for the determinantal representation of $\left(\mathbf{A}^{k+1}\right)^{\dagger}$ in (37).

Taking into account the representations (38)-(39), we derive the determinantal representations of the right and left core inverse matrices that have simpler expressions than those obtained in Theorems 2 and 3.

Corollary 3. Let $\mathbf{A} \in \mathbb{C}_{s}^{n \times n}$ and Ind $\mathbf{A}=1$, and there exist $\mathbf{A}^{\boxplus}$ and $\mathbf{A}_{\circledast}$. Then, $\mathbf{A}^{\boxplus}=\left(a_{i j}^{\boxplus r}\right)$ and $\mathbf{A}_{\circledast}=\left(a_{i j}^{\boxplus l}\right)$ can be expressed as follows: 


$$
\begin{aligned}
& a_{i j}^{\oplus, r}=\frac{\sum_{\alpha \in I_{s, n}\{j\}}\left|\left(\mathbf{A}^{2}\left(\mathbf{A}^{2}\right)^{*}\right)_{j .}\left(\widehat{\mathbf{a}}_{i .}\right)\right|_{\alpha}^{\alpha}}{\sum_{\alpha \in I_{s, n}}\left|\mathbf{A}^{2}\left(\mathbf{A}^{2}\right)^{*}\right|_{\alpha}^{\alpha}}, \\
& a_{i j}^{\oplus, l}=\frac{\sum_{\beta \in J_{s, n}\{i\}}\left|\left(\left(\mathbf{A}^{2}\right)^{*} \mathbf{A}^{2}\right)_{. i}\left(\check{\mathbf{a}}_{. j}\right)\right|_{\beta}^{\beta}}{\sum_{\beta \in J_{s, n}}\left|\left(\mathbf{A}^{2}\right)^{*} \mathbf{A}^{2}\right|_{\beta}^{\beta}},
\end{aligned}
$$

where $\widehat{a}_{i}$ is the ith row of $\widehat{\mathbf{A}}=\mathbf{A}\left(\mathbf{A}^{2}\right)^{*}$ and $\check{\mathbf{a}}_{. j}$ is the $j$ th column of $\check{\mathbf{A}}=\left(\mathbf{A}^{2}\right)^{*} \mathbf{A}$.

\section{Determinantal Representations of the DMP and MPD Inverses}

The concept of the DMP inverse in complex matrices was introduced in [6] by Malik and Thome.

Definition 6. [6] Suppose $\mathbf{A} \in \mathbb{C}^{n \times n}$ and IndA $=k$. A matrix $\mathbf{X} \in \mathbb{C}^{n \times n}$ is said to be the DMP inverse of $\mathbf{A}$ if it satisfies the conditions

$$
\begin{aligned}
\mathbf{X A X} & =\mathbf{X}, \\
\mathbf{X A} & =\mathbf{A}^{d} \mathbf{A}, \\
\mathbf{A}^{k} \mathbf{X} & =\mathbf{A}^{k} \mathbf{A}^{\dagger} .
\end{aligned}
$$

It is denoted by $\mathbf{A}^{d, \dagger}$.

According to [6], if an arbitrary matrix satisfies the system of equations (45), then it is unique and has the following representation:

$$
\mathbf{A}^{d \dagger}=\mathbf{A}^{d} \mathbf{A A}^{\dagger}
$$

Theorem 5. Let $\mathbf{A} \in \mathbb{C}_{s}^{n \times n}$, Ind $\mathbf{A}=k$, and $r k\left(\mathbf{A}^{k}\right)=s_{1}$. Then, its DMP inverse $\mathbf{A}^{d, \dagger}=\left(a_{i j}^{d, \dagger}\right)$ has the following determinantal representations:

$$
\begin{aligned}
a_{i j}^{d, \dagger} & =\frac{\sum_{\alpha \in I_{s, n}\{j\}}\left|\left(\mathbf{A A}^{*}\right)_{j .}\left(\mathbf{u}_{i .}^{(1)}\right)\right|_{\alpha}^{\alpha}}{\sum_{\beta \in J_{s_{1}, n}}\left|\mathbf{A}^{k+1}\right|_{\beta}^{\beta} \sum_{\alpha \in I_{s, n}}\left|\mathbf{A} \mathbf{A}^{*}\right|_{\alpha}^{\alpha}} \\
& =\frac{\sum_{\beta \in J_{s_{1}, n}\{i\}}\left|\left(\mathbf{A}^{k+1}\right)_{. i}\left(\mathbf{u}_{. j}^{(2)}\right)\right|_{\beta}^{\beta}}{\sum_{\beta \in J_{s_{1}, n}}\left|\mathbf{A}^{k+1}\right|_{\beta}^{\beta} \sum_{\beta \in J_{s, n}}\left|\mathbf{A A}^{*}\right|_{\beta}^{\beta}},
\end{aligned}
$$

where

$$
\begin{aligned}
& \mathbf{u}_{i .}^{(1)}=\left[\sum_{\beta \in J_{s_{1}, n}\{i\}}\left|\left(\mathbf{A}^{k+1}\right)_{. i}\left(\widetilde{\mathbf{a}}_{. f}\right)\right|_{\beta}^{\beta}\right] \in \mathbb{C}^{1 \times n}, \quad f=1, \ldots, n, \\
& \mathbf{u}_{. j}^{(2)}=\left[\sum_{\alpha \in I_{s, n}\{j\}}\left|\left(\mathbf{A A}^{*}\right)_{j .}\left(\widetilde{\mathbf{a}}_{. l}\right)\right|_{\alpha}^{\alpha}\right] \in \mathbb{C}^{n \times 1}, \quad l=1, \ldots, n .
\end{aligned}
$$

Here, $\widetilde{\mathbf{a}}_{f}$ and $\widetilde{\mathbf{a}}_{l}$ are the fth column and the lth row of $\widetilde{\mathbf{A}}:=\mathbf{A}^{k+1} \mathbf{A}^{*}$.
Proof. Taking into account (46) for $\mathbf{A}^{d, \dagger}$, we get

$$
a_{i j}^{d, \dagger}=\sum_{l=1}^{n} \sum_{f=1}^{n} a_{i l}^{d} a_{l f} a_{f j}^{\dagger}
$$

By substituting (14) and (10) for the determinantal representations of $\mathbf{A}^{d}$ and $\mathbf{A}^{\dagger}$ in (49), we get

$$
\begin{aligned}
a_{i j}^{d, \dagger}= & \sum_{l=1}^{n} \sum_{f=1}^{n} \frac{\sum_{\beta \in J_{s_{1}, n}\{i\}}\left|\left(\mathbf{A}^{k+1}\right)_{. i}\left(\mathbf{a}_{. l}^{(k)}\right)\right|_{\beta}^{\beta}}{\sum_{\beta \in J_{s_{1}, n}}\left|\mathbf{A}^{k+1}\right|_{\beta}^{\beta}} a_{l f} \\
& \cdot \frac{\sum_{\alpha \in I_{s, n}\{j\}}\left|\left(\mathbf{A A}^{*}\right)_{j .}\left(\mathbf{a}_{f .}^{*}\right)\right|_{\alpha}^{\alpha}}{\sum_{\alpha \in I_{s, n}}\left|\mathbf{A A}^{*}\right|_{\alpha}^{\alpha}} \\
= & \sum_{l=1}^{n} \sum_{f=1}^{n} \frac{\sum_{s_{1}, n}\left|\left(\mathbf{A}^{k+1}\right)_{. i}\left(\mathbf{e}_{. l}\right)\right|_{\beta}^{\beta}}{\sum_{\beta \in J_{s_{1}, n}}\left|\mathbf{A}^{k+1}\right|_{\beta}^{\beta}} \frac{\sum_{\alpha \in I_{s, n}\{j\}}\left|\left(\mathbf{A A}^{*}\right)_{j .}\left(\mathbf{e}_{f .}\right)\right|_{\alpha}^{\alpha}}{\sum_{\alpha \in I_{s, n}}\left|\mathbf{A A}^{*}\right|_{\alpha}^{\alpha}},
\end{aligned}
$$

where $\mathbf{e}_{l}$ and $\mathbf{e}_{l}$ are the $l$ th unit column and row vectors and $\tilde{a}_{l f}$ is the (lf)th element of the matrix $\widetilde{\mathbf{A}}=\mathbf{A}^{k+1} \mathbf{A}^{*}$. If we put

$$
\begin{aligned}
u_{i f}^{(1)} & :=\sum_{l=1}^{n} \sum_{\beta \in J_{s_{1}, n}\{i\}}\left|\left(\mathbf{A}^{k+1}\right)_{. i}\left(\mathbf{e}_{. l}\right)\right|_{\beta}^{\beta} \widetilde{a}_{l f} \\
& =\sum_{\beta \in J_{s_{1}, n}\{i\}}\left|\left(\mathbf{A}^{k+1}\right)_{. i}\left(\widetilde{\mathbf{a}}_{. f}\right)\right|_{\beta}^{\beta},
\end{aligned}
$$

as the $f$ th component of the row vector $\mathbf{u}_{i .}^{(1)}=\left[u_{i 1}^{(1)}\right.$, $\left.\ldots, u_{i n}^{(1)}\right]$. Then from

$$
\sum_{f=1}^{n} u_{i f}^{(1)} \sum_{\alpha \in I_{s, n}\{j\}}\left|\left(\mathbf{A A}^{*}\right)_{j .}\left(\mathbf{e}_{f .}\right)\right|_{\alpha}^{\alpha}=\sum_{\alpha \in I_{s, n}\{j\}}\left|\left(\mathbf{A A}^{*}\right)_{j .}\left(\mathbf{u}_{i .}^{(1)}\right)\right|_{\alpha}^{\alpha},
$$

it follows (47). If we initially obtain

$$
\begin{aligned}
u_{l j}^{(2)} & :=\sum_{f=1}^{n} \tilde{a}_{l f} \sum_{\alpha \in I_{s, n}\{j\}}\left|\left(\mathbf{A} \mathbf{A}^{*}\right)_{j .}\left(\mathbf{e}_{f .}\right)\right|_{\alpha}^{\alpha} \\
& =\sum_{\alpha \in I_{s, n}\{j\}}\left|\left(\mathbf{A A}^{*}\right)_{j .}\left(\widetilde{\mathbf{a}}_{l .}\right)\right|_{\alpha}^{\alpha}
\end{aligned}
$$

the $l$ th component of the column vector $\mathbf{u}_{. j}^{(2)}=\left[u_{1 j}^{(2)}\right.$, $\left.\ldots, u_{n j}^{(2)}\right]$. Then from

$$
\sum_{l=1}^{n} \sum_{\beta \in J_{s_{1}, n}\{i\}}\left|\left(\mathbf{A}^{k+1}\right)_{. i}\left(\mathbf{e}_{. l}\right)\right|_{\beta}^{\beta} u_{l j}^{(2)}=\sum_{\beta \in J_{s_{1}, n}\{i\}}\left|\left(\mathbf{A}^{k+1}\right)_{. i}\left(\mathbf{u}_{. j}^{(2)}\right)\right|_{\beta}^{\beta}
$$

it follows (47).

The name of the DMP inverse is in accordance with the order of using the Drazin inverse (D) and the Moore- 
Penrose (MP) inverse. In that connection, it would be logical to consider the following definition.

Definition 7. Suppose $\mathbf{A} \in \mathbb{C}^{n \times n}$ and $\operatorname{Ind} \mathbf{A}=k$. A matrix $\mathbf{X} \in \mathbb{C}^{n \times n}$ is said to be the MPD inverse of $\mathbf{A}$ if it satisfies the conditions

$$
\begin{aligned}
\mathbf{X A X} & =\mathbf{X}, \\
\mathbf{A X} & =\mathbf{A A}^{d}, \\
\mathbf{X A}^{k} & =\mathbf{A}^{\dagger} \mathbf{A}^{k} .
\end{aligned}
$$

It is denoted by $\mathbf{A}^{\dagger, d}$.

Similar as for the DMP inverse, it can be proved that the matrix $\mathbf{A}^{\dagger, d}$ is unique, and it can be represented as

$$
\mathbf{A}^{\dagger, d}=\mathbf{A}^{\dagger} \mathbf{A} \mathbf{A}^{d}
$$

Theorem 6. Let $\mathbf{A} \in \mathbb{C}_{s}^{n \times n}$, Ind $\mathbf{A}=k$, and $r k \mathbf{A}^{k}=s_{1}$. Then, its MPD inverse $\mathbf{A}^{\dagger, d}=\left(a_{i j}^{\dagger, d}\right)$ has the following determinantal representations:

$$
a_{i j}^{\dagger, d}=\frac{\sum_{\beta \in J_{s, n}\{i\}}\left|\left(\mathbf{A}^{*} \mathbf{A}\right)_{. i}\left(\mathbf{v}_{. j}^{(1)}\right)\right|_{\beta}^{\beta}}{\sum_{\beta \in J_{s, n}}\left|\mathbf{A}^{*} \mathbf{A}\right|_{\beta}^{\beta} \sum_{\beta \in I_{s_{1}, n}}\left|\mathbf{A}^{k+1}\right|_{\alpha}^{\alpha}}=\frac{\sum_{\alpha \in I_{s_{1}, n}}\{j\}\left|\left(\mathbf{A}^{k+1}\right)_{j .}\left(\mathbf{v}_{i .}^{(2)}\right)\right|_{\alpha}^{\alpha}}{\sum_{\alpha \in I_{s_{1}, n}}\left|\mathbf{A}^{*} \mathbf{A}\right|_{\beta}^{\beta} \sum_{\alpha \in I_{s, n}}\left|\mathbf{A}^{k+1}\right|_{\alpha}^{\alpha},}
$$

where

$$
\begin{aligned}
& \mathbf{v}_{. j}^{(1)}=\left[\sum_{\alpha \in I_{s_{1}, n}\{j\}}\left|\left(\mathbf{A}^{k+1}\right)_{j .}\left(\widehat{\mathbf{a}}_{l .}\right)\right|_{\alpha}^{\alpha}\right] \in \mathbb{C}^{n \times 1}, \quad l=1, \ldots, n, \\
& \mathbf{v}_{i .}^{(2)}=\left[\sum_{\beta \in J_{s, n}\{i\}}\left|\left(\mathbf{A}^{*} \mathbf{A}\right)_{. i}\left(\widehat{\mathbf{a}}_{\cdot f}\right)\right|_{\beta}^{\beta}\right] \in \mathbb{C}^{1 \times n}, \quad l=1, \ldots, n .
\end{aligned}
$$

Here, $\widehat{\mathbf{a}}_{l .}$ and $\widehat{\mathbf{a}}_{\text {.f }}$ are the lth row and the fth column of $\widehat{\mathbf{A}}:=\mathbf{A}^{*} \mathbf{A}^{k+1}$.

Proof. The proof is similar to the proof of Theorem 6 .

\section{Determinantal Representations of the CMP Inverse}

Definition 8 [7]. Suppose $\mathbf{A} \in \mathbb{C}^{n \times n}$ has the core-nilpotent decomposition $\mathbf{A}=\mathbf{A}_{1}+\mathbf{A}_{2}$, where Ind $\mathbf{A}_{1}=\operatorname{Ind} \mathbf{A}, \mathbf{A}_{2}$ is nilpotent, and $\mathbf{A}_{1} \mathbf{A}_{2}=\mathbf{A}_{2} \mathbf{A}_{1}=0$. The CMP inverse of $\mathbf{A}$ is called the matrix $\mathbf{A}^{c, \dagger}:=\mathbf{A}^{\dagger} \mathbf{A}_{1} \mathbf{A}^{\dagger}$.
Lemma 4 [7]. Let $\mathbf{A} \in \mathbb{C}^{n \times n}$. The matrix $\mathbf{X}=\mathbf{A}^{c, \dagger}$ is the unique matrix that satisfies the following system of equations:

$$
\begin{aligned}
\mathbf{X A X} & =\mathbf{X}, \\
\mathbf{A X A} & =\mathbf{A}_{1}, \\
\mathbf{A X} & =\mathbf{A}_{1} \mathbf{A}^{\dagger}, \\
\mathbf{X A} & =\mathbf{A}^{\dagger} \mathbf{A}_{1} .
\end{aligned}
$$

Moreover,

$$
\mathbf{A}^{c, \dagger}=\mathbf{Q}_{A} \mathbf{A}^{d} \mathbf{P}_{A} .
$$

Taking into account (60), it follows the next theorem about determinantal representations of the quaternion CMP inverse.

Theorem 7. Let $\mathbf{A} \in \mathbb{C}_{s}^{n \times n}$, Ind $\mathbf{A}=m$, and $r k\left(\mathbf{A}^{m}\right)=s_{1}$. Then, the determinantal representations of its CMP inverse $\mathbf{A}^{c, \dagger}=\left(a_{i j}^{c, \dagger}\right)$ can be expressed as

$$
\begin{aligned}
a_{i j}^{c, \dagger} & =\frac{\sum_{\beta \in J_{s, n}\{i\}}\left|\left(\mathbf{A}^{*} \mathbf{A}\right)_{. i}\left(\mathbf{v}_{. j}^{(l)}\right)\right|_{\beta}^{\beta}}{\left(\sum_{\beta \in J_{s, n}}\left|\mathbf{A}^{*} \mathbf{A}\right|_{\beta}^{\beta}\right)^{2} \sum_{\beta \in J_{s_{1}, n}}\left|\mathbf{A}^{m+1}\right|_{\beta}^{\beta}} \\
& =\frac{\sum_{\alpha \in I_{s, n}\{j\}}\left|\left(\mathbf{A A}^{*}\right)_{j .}\left(\mathbf{w}_{i .}^{(l)}\right)\right|_{\alpha}^{\alpha}}{\left(\sum_{\alpha \in I_{s, n}}\left|\mathbf{A} \mathbf{A}^{*}\right|_{\alpha}^{\alpha}\right)^{2} \sum_{\beta \in J_{s_{1}, n}}\left|\mathbf{A}^{m+1}\right|_{\beta}^{\beta}},
\end{aligned}
$$

for all $l=1,2$, where

$$
\begin{aligned}
& \mathbf{v}_{. j}^{(1)}=\left[\sum_{\alpha \in I_{s, n}\{j\}}\left|\left(\mathbf{A A}^{*}\right)_{j .}\left(\widehat{u}_{t}\right)\right|_{\alpha}^{\alpha}\right] \in \mathbb{C}^{n \times 1}, \quad t=1, \ldots, n, \\
& \mathbf{w}_{i .}^{(1)}=\left[\sum_{\beta \in J_{s, n}\{i\}}\left|\left(\mathbf{A}^{*} \mathbf{A}\right)_{. i}\left(\widehat{u}_{. k}\right)\right|_{\beta}^{\beta}\right] \in \mathbb{C}^{1 \times n}, \quad k=1, \ldots, n, \\
& \mathbf{v}_{. j}^{(2)}=\left[\sum_{\alpha \in I_{s, n}\{j\}}\left|\left(\mathbf{A}^{*} \mathbf{A}\right)_{j .}\left(\widetilde{g}_{t .}\right)\right|_{\alpha}^{\alpha}\right] \in \mathbb{C}^{n \times 1}, \quad t=1, \ldots, n, \\
& \mathbf{w}_{i .}^{(2)}=\left[\sum_{\beta \in J_{s, n}\{i\}}\left|\left(\mathbf{A}^{*} \mathbf{A}\right)_{. i}\left(\widetilde{g}_{. k}\right)\right|_{\beta}^{\beta}\right] \in \mathbb{C}^{1 \times n}, \quad k=1, \ldots, n .
\end{aligned}
$$

Here, $\widehat{\mathbf{u}}_{t .}$ is the th row and $\widehat{\mathbf{u}}_{. k}$ is the kth column of $\widehat{\mathbf{U}}:=\mathbf{U A A}^{*}, \widetilde{\mathbf{g}}_{t}$ is the th row and $\widetilde{\mathbf{g}}_{. k}$ is the kth column of 
$\widetilde{\mathbf{G}}:=\mathbf{A}^{*} \mathbf{A G}$, and the matrices $\mathbf{U}=\left(u_{i j}\right) \in \mathbb{C}^{n \times n}$ and $\mathbf{G}=\left(g_{i j}\right) \in \mathbb{C}^{n \times n}$ are such that

$$
\begin{aligned}
& u_{i j}=\sum_{\alpha \in I_{s_{1}, n}\{j\}}\left|\left(\mathbf{A}^{m+1}\right)_{j .}\left(\widehat{\mathbf{a}}_{i .}\right)\right|_{\alpha}^{\alpha}, \\
& g_{i j}=\sum_{\beta \in J_{s_{1}, n}\{i\}}\left|\left(\mathbf{A}^{m+1}\right)_{. i}\left(\widetilde{\mathbf{a}}_{. j}\right)\right|_{\beta}^{\beta},
\end{aligned}
$$

where $\widehat{a}_{i .}$ is the ith row of $\widehat{A}:=\mathbf{A}^{*} \mathbf{A}^{m+1}$ and $\widetilde{a}_{. j}$ is the $j$ th column of $\widetilde{A}:=\mathbf{A}^{m+1} \mathbf{A}^{*}$.

Proof. Suppose $\mathbf{Q}_{A}=\left(q_{i l}^{A}\right), \mathbf{A}^{d}=\left(a_{i l}^{d}\right)$, and $\mathbf{P}_{A}=\left(p_{i l}^{A}\right)$. Taking into account (60), we get

$$
a_{i j}^{c, \dagger}=\sum_{l=1}^{n} \sum_{k=1}^{n} q_{i l}^{A} a_{l k}^{d} p_{k j}^{A}
$$

(a) Taking into account the expressions (14), (12), and (13) for the determinantal representations of $\mathbf{A}^{d}, \mathbf{Q}_{A}$, and $\mathbf{P}_{A}$, respectively, we have

$$
\begin{aligned}
a_{i j}^{c, \dagger}= & \sum_{l} \sum_{t} \frac{\sum_{\beta \in J_{s, n}\{i\}}\left|\left(\mathbf{A}^{*} \mathbf{A}\right)_{. i}\left(\dot{a}_{. t}\right)\right|_{\beta}^{\beta}}{\sum_{\beta \in J_{s, n}}\left|\mathbf{A}^{*} \mathbf{A}\right|_{\beta}^{\beta}} \\
& \cdot \frac{\sum_{\alpha \in I_{s_{1}, n}\{l\}}\left|\left(\mathbf{A}^{m+1}\right)_{l .}\left(\mathbf{a}_{t .}^{(m)}\right)\right|_{\alpha}^{\alpha}}{\sum_{\alpha \in I_{s_{1}, n}}\left|\mathbf{A}^{m+1}\right|_{\alpha}^{\alpha}} \\
& \times \frac{\sum_{\alpha \in I_{s, n}\{j\}}\left|\left(\mathbf{A} \mathbf{A}^{*}\right)_{j .}\left(\ddot{\mathbf{a}}_{l .}\right)\right|_{\alpha}^{\alpha}}{\sum_{\alpha \in I_{s, n}}\left|\mathbf{A} \mathbf{A}^{*}\right|_{\alpha}^{\alpha}}
\end{aligned}
$$

where $\dot{\mathbf{a}}_{. t}$ is the $t$ th column of $\mathbf{A}^{*} \mathbf{A}, \ddot{\mathbf{a}}_{l}$ is the $l$ th row of $\mathbf{A} \mathbf{A}^{*}$, and $\mathbf{a}_{t}^{(m)}$ is the $t$ th row of $\mathbf{A}^{m}$. So, it is clear that

$$
\begin{aligned}
a_{i j}^{c, \dagger}= & \sum_{l} \sum_{t} \sum_{k} \\
& \cdot \frac{\sum_{\beta \in J_{s, n}\{i\}}\left|\left(\mathbf{A}^{*} \mathbf{A}\right)_{. i}\left(\mathbf{e}_{. t}\right)\right|_{\beta}^{\beta} \widehat{a}_{t k} \sum_{\alpha \in I_{s_{1}, n}\{l\}}\left|\left(\mathbf{A}^{m+1}\right)_{l .}\left(\mathbf{e}_{k .}\right)\right|_{\alpha}^{\alpha}}{\sum_{\beta \in J_{s, n}}\left|\mathbf{A}^{*} \mathbf{A}\right|_{\beta}^{\beta} \sum_{\alpha \in I_{s_{1}, n}}\left|\mathbf{A}^{m+1}\right|_{\alpha}^{\alpha}} \\
& \times \frac{\sum_{\alpha \in I_{s, n}\{j\}}\left|\left(\mathbf{A A}^{*}\right)_{j .}\left(\ddot{\mathbf{a}}_{l .}\right)\right|_{\alpha}^{\alpha}}{\sum_{\alpha \in I_{s, n}}\left|\mathbf{A A}^{*}\right|_{\alpha}^{\alpha}}
\end{aligned}
$$

where $\mathbf{e}_{. t}$ is the $t$ th unit column vector, $\mathbf{e}_{k}$. is the $k$ th unit row vector, and $\hat{a}_{t k}$ is the $(t k)$ th element of $\widehat{\mathbf{A}}=\mathbf{A}^{*} \mathbf{A}^{m+1}$. Denote

$$
\begin{aligned}
u_{t l} & :=\sum_{k} \widehat{a}_{t k} \sum_{\alpha \in I_{s_{1}, n}\{j\}}\left|\left(\mathbf{A}^{m+1}\right)_{l .}\left(\mathbf{e}_{k .}\right)\right|_{\alpha}^{\alpha} \\
& =\sum_{\alpha \in I_{s_{1}, n}\{j\}}\left|\left(\mathbf{A}^{m+1}\right)_{l .}\left(\widehat{a}_{t .}\right)\right|_{\alpha}^{\alpha},
\end{aligned}
$$

the $t$ th component of a column-vector $\mathbf{u}_{. l}=\left[u_{1 l}, \ldots\right.$, $\left.u_{n l}\right]$. Since

$$
\sum_{t} \sum_{\beta \in J_{s, n}\{i\}}\left|\left(\mathbf{A}^{*} \mathbf{A}\right)_{. i}\left(\mathbf{e}_{. t}\right)\right|_{\beta}^{\beta} u_{t l}=\sum_{\beta \in J_{s, n}\{i\}}\left|\left(\mathbf{A}^{*} \mathbf{A}\right)_{. i}\left(\mathbf{u}_{. l}\right)\right|_{\beta}^{\beta},
$$

then

$$
a_{i j}^{c, \dagger}=\sum_{l} \frac{\sum_{\beta \in J_{s, n}\{i\}}\left|\left(\mathbf{A}^{*} \mathbf{A}\right)_{. i}\left(\mathbf{u}_{l l}\right)\right|_{\beta}^{\beta} \sum_{\alpha \in I_{s, n}\{j\}}\left|\left(\mathbf{A A}^{*}\right)_{j .}\left(\ddot{\mathbf{a}}_{l .}\right)\right|_{\alpha}^{\alpha}}{\sum_{\beta \in J_{r, n}}\left|\mathbf{A}^{*} \mathbf{A}\right|_{\beta}^{\beta} \sum_{\alpha \in I_{s_{1}, n}}\left|\mathbf{A}^{m+1}\right|_{\alpha}^{\alpha} \sum_{\alpha \in I_{s, n}}\left|\mathbf{A} \mathbf{A}^{*}\right|_{\alpha}^{\alpha}} .
$$

Construct the matrix $\mathbf{U}=\left(u_{t l}\right) \in \mathbb{C}^{n \times n}$, where $u_{t l}$ is given by (70), and denote $\widehat{\mathbf{U}}:=\mathbf{U A A}^{*}$. Then, taking into account that $\left|\mathbf{A}^{*} \mathbf{A}\right|_{\beta}^{\beta}=\left|\mathbf{A} \mathbf{A}^{*}\right|_{\alpha}^{\alpha}$, we have

$$
a_{i j}^{c, \dagger}=\frac{\sum_{t} \sum_{k} \sum_{\beta \in J_{s, n}\{i\}}\left|\left(\mathbf{A}^{*} \mathbf{A}\right)_{. i}\left(\mathbf{e}_{. t}\right)\right|_{\beta}^{\beta} \widehat{u}_{t k} \sum_{\alpha \in I_{s, n}}\{j\}\left|\left(\mathbf{A A}^{*}\right)_{j .}\left(\mathbf{e}_{k .}\right)\right|_{\alpha}^{\alpha}}{\left(\sum_{\beta \in J_{s, n}}\left|\mathbf{A}^{*} \mathbf{A}\right|_{\beta}^{\beta}\right)^{2} \sum_{\alpha \in I_{s_{1}, n}}\left|\mathbf{A}^{m+1}\right|_{\alpha}^{\alpha}} .
$$

If we put that

$$
\begin{aligned}
v_{t j}^{(1)} & :=\sum_{k} \widehat{u}_{t k} \sum_{\alpha \in I_{s, n}\{j\}}\left|\left(\mathbf{A A}^{*}\right)_{j .}\left(\mathbf{e}_{k .}\right)\right|_{\alpha}^{\alpha} \\
& =\sum_{\alpha \in I_{s, n}\{j\}}\left|\left(\mathbf{A A}^{*}\right)_{j .}\left(\widehat{u}_{t .}\right)\right|_{\alpha}^{\alpha},
\end{aligned}
$$

is the $t$ th component of a column vector, $\mathbf{v}_{. j}^{(1)}=\left[v_{1 j}^{(1)}\right.$, $\left.\ldots, v_{n j}^{(1)}\right]$. Then from

$$
\sum_{t} \sum_{\beta \in J_{s, n}\{i\}}\left|\left(\mathbf{A}^{*} \mathbf{A}\right)_{. i}\left(\mathbf{e}_{. t}\right)\right|_{\beta}^{\beta} v_{t j}^{(1)}=\sum_{\beta \in J_{s, n}\{i\}}\left|\left(\mathbf{A}^{*} \mathbf{A}\right)_{. i}\left(\mathbf{v}_{. j}^{(1)}\right)\right|_{\beta}^{\beta},
$$

it follows (61) with $\mathbf{v}_{. j}^{(1)}$ given by (62). If we initially put

$$
\begin{aligned}
w_{i k}^{(1)} & :=\sum_{t} \sum_{\beta \in J_{s, n}\{i\}}\left|\left(\mathbf{A}^{*} \mathbf{A}\right)_{. i}\left(\mathbf{e}_{. t}\right)\right|_{\beta}^{\beta} \widehat{u}_{t k} \\
& =\sum_{\beta \in J_{s, n}\{i\}}\left|\left(\mathbf{A}^{*} \mathbf{A}\right)_{. i}\left(\widehat{u}_{. k}\right)\right|_{\beta}^{\beta},
\end{aligned}
$$


as the $k$ th component of the row vector, $\mathbf{w}_{i .}^{(1)}=\left[w_{i 1}^{(1)}\right.$, $\left.\ldots, w_{\text {in }}^{(1)}\right]$. Then from

$\sum_{k} w_{i k}^{(1)} \sum_{\alpha \in I_{s, n}\{j\}}\left|\left(\mathbf{A}^{2}\right)_{j .}\left(\mathbf{e}_{k .}\right)\right|_{\alpha}^{\alpha}=\sum_{\alpha \in I_{s, n}\{j\}}\left|\left(\mathbf{A}^{2}\right)_{j .} .\left(\mathbf{w}_{i .}^{(1)}\right)\right|_{\alpha}^{\alpha}$,

it follows (61) with $\mathbf{w}_{i .}^{(1)}$ given by (64).

(b) By using the determinantal representation (14) for $\mathbf{A}^{d}$ in (67), we have

$$
\begin{aligned}
a_{i j}^{c, \dagger}= & \sum_{k} \sum_{t} \frac{\sum_{\beta \in J_{s, n}\{i\}}\left|\left(\mathbf{A}^{*} \mathbf{A}\right)_{. i}\left(\dot{a}_{. t}\right)\right|_{\beta}^{\beta}}{\sum_{\beta \in J_{s, n}}\left|\mathbf{A}^{*} \mathbf{A}\right|_{\beta}^{\beta}} \\
& \cdot \frac{\sum_{\beta \in J_{s, n}\{t\}}\left|\left(\mathbf{A}^{m+1}\right)_{. t}\left(\mathbf{a}_{. k}^{(m)}\right)\right|_{\beta}^{\beta}}{\sum_{\beta \in J_{s, n}}\left|\mathbf{A}^{m+1}\right|_{\beta}^{\beta}} \\
& \times \frac{\sum_{\alpha \in I_{s, n}\{j\}}\left|\left(\mathbf{A A}^{*}\right)_{j .}\left(\ddot{\mathbf{a}}_{k .}\right)\right|_{\alpha}^{\alpha}}{\sum_{\alpha \in I_{s, n}}\left|\mathbf{A} \mathbf{A}^{*}\right|_{\alpha}^{\alpha}}
\end{aligned}
$$

Therefore,

$$
\begin{aligned}
a_{i j}^{c, \dagger}= & \sum_{l} \sum_{k} \sum_{t} \frac{\sum_{\beta \in J_{s, n}\{i\}}\left(\left(\mathbf{A}^{*} \mathbf{A}\right)_{. i}\left(\dot{a}_{. t}\right)\right)_{\beta}^{\beta}}{\sum_{\beta \in I_{s, n}}\left|\mathbf{A}^{*} \mathbf{A}\right|_{\beta}^{\beta}} \\
& \times \frac{\sum_{\beta \in \epsilon_{s_{1, n}}\{t\}}\left|\left(\mathbf{A}^{m+1}\right)_{. t}\left(\mathbf{e}_{. k}\right)\right|_{\beta}^{\beta}}{\sum_{\beta \in J_{s, n} \mid}\left|\mathbf{A}^{m+1}\right|_{\beta}^{\beta}} \\
& \cdot \tilde{a}_{k l} \frac{\sum_{\alpha \in I_{s, n}\{j\}}\left|\left(\mathbf{A A}^{*}\right)_{j .}\left(\mathbf{e}_{l .}\right)\right|_{\alpha}^{\alpha}}{\sum_{\alpha \in I_{s, n}}\left|\mathbf{A A}^{*}\right|_{\alpha}^{\alpha}}
\end{aligned}
$$

$$
\left.=\sum_{\beta \in J_{s_{1}, n}\{t\}} \mid\left(\mathbf{A}^{m+1}\right)\right)\left._{. t}\left(\widetilde{\mathbf{a}}_{l}\right)\right|_{\beta^{\prime}} ^{\beta},
$$

the $l$ th component of a row-vector $\mathbf{g}_{t .}=\left[g_{t 1}, \ldots, g_{t n}\right]$. Then,

$$
\begin{gathered}
\sum_{l} g_{t l} \sum_{\alpha \in I_{s, n}\{j\}}\left|\left(\mathbf{A A}^{*}\right)_{j .}\left(\mathbf{e}_{l .}\right)\right|_{\alpha}^{\alpha} \\
=\sum_{\alpha \in I_{s, n}\{j\}}\left|\left(\mathbf{A A}^{*}\right)_{j .}\left(\mathbf{g}_{t .}\right)\right|_{\alpha}^{\alpha} .
\end{gathered}
$$

From this, it follows that

$$
a_{i j}^{c, \dagger}=\sum_{t} \frac{\sum_{\beta \in I_{s, n}\{i\}}\left|\left(\mathbf{A}^{*} \mathbf{A}\right)_{. i}\left(\dot{a}_{. t}\right)\right|_{\beta}^{\beta} \sum_{\alpha \in I_{s, n}\{j\}}\left(\left|\mathbf{A A}^{*}\right|_{j .}\left(\mathbf{g}_{t .}\right)\right)_{\alpha}^{\alpha}}{\sum_{\beta \in J_{r, n}}\left|\mathbf{A}^{*} \mathbf{A}\right|_{\beta}^{\beta} \sum_{\alpha \in I_{s, n} \mid}\left|\mathbf{A}^{m+1}\right|_{\alpha}^{\alpha} \sum_{\alpha \in I_{s, n}}\left|\mathbf{A A}^{*}\right|_{\alpha}^{\alpha}} .
$$

Construct the matrix $\mathbf{G}=\left(g_{t l}\right) \in \mathbb{C}^{n \times n}$, where $g_{t l}$ is given by (80). Denote $\widetilde{G}:=\mathbf{A}^{*}$ AG. Then

$$
a_{i j}^{c, \dagger}=\frac{\sum_{t} \sum_{k} \sum_{\beta \in I_{s, n}\{i\}}\left|\left(\mathbf{A}^{*} \mathbf{A}\right)_{. i}\left(\mathbf{e}_{t .}\right)\right|_{\beta}^{\beta} \widehat{u}_{t k} \sum_{\alpha \in I_{s, n}\{j\}}\left|\left(\mathbf{A A}^{*}\right)_{j .}\left(\mathbf{e}_{k .}\right)\right|_{\alpha}^{\alpha}}{\left(\sum_{\beta \in J_{s, n}}\left|\mathbf{A}^{*} \mathbf{A}\right|_{\beta}^{\beta}\right)^{2} \sum_{\alpha \in I_{I_{1}, n}}\left|\mathbf{A}^{m+1}\right|_{\alpha}^{\alpha}} .
$$

Similar to the above, if we denote by

$v_{t j}^{(1)}:=\sum_{k} \widehat{u}_{t k} \sum_{\alpha \in I_{s, n}\{j\}}\left|\left(\mathbf{A A}^{*}\right)_{j .}\left(\mathbf{e}_{k} .\right)\right|_{\alpha}^{\alpha}=\sum_{\alpha \in I_{s, n}\{j\}}\left|\left(\mathbf{A A}^{*}\right)_{j .} \cdot\left(\widehat{\mathbf{u}}_{t .}\right)\right|_{\alpha^{\prime}}^{\alpha}$,

the th component of a column vector $\mathbf{v}_{. j}^{(2)}=\left[v_{1 j}^{(2)}\right.$, $\left.\ldots, v_{n j}^{(2)}\right]$, then

$\sum_{t} \sum_{\beta \in J_{s, n}\{i\}}\left|\left(\mathbf{A}^{*} \mathbf{A}\right)_{. i}\left(\mathbf{e}_{. t}\right)\right|_{\beta}^{\beta} v_{t j}^{(1)}=\sum_{\beta \in J_{s, n}\{i\}}\left|\left(\mathbf{A}^{*} \mathbf{A}\right)_{.}\left(\mathbf{v}_{. j}^{(1)}\right)\right|_{\beta}^{\beta}$.

Thus, we have (61) with $\mathbf{v}_{. j}^{(2)}$ given by (64).

If, now, we denote by

$w_{i k}^{(1)}:=\sum_{t} \sum_{\beta \in J_{s, n}\{i\}}\left|\left(\mathbf{A}^{*} \mathbf{A}\right)_{. i}\left(\mathbf{e}_{. t}\right)\right|_{\beta}^{\beta} \widehat{u}_{t k}=\sum_{\beta \in J_{s, n}\{i\}}\left|\left(\mathbf{A}^{*} \mathbf{A}\right)_{. i}\left(\widehat{\mathbf{u}}_{. k}\right)\right|_{\beta}^{\beta}$,

the $k$ th component of the row vector $\mathbf{w}_{i .}^{(1)}=\left[w_{i 1}^{(1)}\right.$, $\left.\ldots, w_{i n}^{(1)}\right]$. Then, from 


$$
\sum_{k} w_{i k}^{(1)} \sum_{\alpha \in I_{s, n}\{j\}}\left|\left(\mathbf{A}^{2}\right)_{j .}\left(\mathbf{e}_{k .}\right)\right|_{\alpha}^{\alpha}=\sum_{\alpha \in I_{s, n}\{j\}}\left|\left(\mathbf{A}^{2}\right)_{j .}\left(\mathbf{w}_{i .}^{(1)}\right)\right|_{\alpha}^{\alpha} .
$$

So, finally, we have (61) with $\mathbf{w}_{i .}^{(2)}$ given by (65).

Remark 6. Concerning the possible existence of dual to the CMP inverse, we note the following. Taking into account $\left(\mathbf{A}^{d}\right)^{2} \mathbf{A}=\mathbf{A}^{d}$,

$$
\mathbf{P}_{A} \mathbf{A}^{d} \mathbf{Q}_{A}=\mathbf{A} \mathbf{A}^{\dagger}\left(\mathbf{A}^{d}\right)^{2} \mathbf{A} \mathbf{A}^{\dagger} \mathbf{A}=\mathbf{A} \mathbf{A}^{\dagger} \mathbf{A}^{d}=\mathbf{A} \mathbf{A}^{\dagger} \mathbf{A}\left(\mathbf{A}^{d}\right)^{2}=\mathbf{A}^{d}
$$

\section{Cramer's Rule for Some Constrained Linear Equations}

Cramer's rules for special solutions to systems of linear equations stand as possible applications of determinantal representations of the core inverse and its generalizations. For instance, consider Cramer's rule for the system which has the important applied significance.

First, note that the core inverse has close relation with the Bott-Duffin inverse. According to [19], the BottDuffin inverse of $\mathbf{A} \in \mathbb{T}^{n \times n}$ with respect to $\mathscr{C}(\mathbf{A})$ can be given by

$$
\mathbf{A}_{\mathscr{C}(\mathbf{A})}^{(-1)}=\mathbf{P}_{A}\left[\left(\mathbf{A}-\mathbf{I}_{n}\right) \mathbf{P}_{A}+\mathbf{I}_{n}\right]^{-1},
$$

where $\left(\mathbf{A}-\mathbf{I}_{n}\right) \mathbf{P}_{A}+\mathbf{I}_{n}$ needs to be nonsingular. According to [1], $\mathbf{A}_{\mathscr{C}(\mathbf{A})}^{(-1)}$ coincides with the right core inverse $\mathbf{A}^{\boxplus}$. One can find more details regarding the Bott-Duffin inverse in [44].

Consider the constrained linear equations

$$
\mathbf{A x}+\mathbf{y}=\mathbf{b}, \quad \mathbf{x} \in \mathscr{C}(\mathbf{A}), \mathbf{y} \in \mathscr{N}(\mathbf{A}),
$$

where $\mathbf{A} \in \mathbb{C}^{n \times n}$ and $\mathbf{b} \in \mathbb{C}^{n}$. According to [45], this equation arises in electrical networks and its solution is determined by the following lemma.

Lemma 5. Let $\left(\mathbf{A}-\mathbf{I}_{n}\right) \mathbf{P}_{A}+\mathbf{I}_{n}$ be nonsingular. Then, equation (90) has for every $\mathbf{b}$ the unique solution

$$
\begin{aligned}
& \mathbf{x}=\mathbf{A}_{\mathscr{C}(\mathbf{A})}^{(-1)} \mathbf{b}, \\
& \mathbf{y}=\left(\mathbf{I}-\mathbf{A} \mathbf{A}_{\mathscr{C}(\mathbf{A})}^{(-1)}\right) \mathbf{b} .
\end{aligned}
$$

The following theorem gives Cramer's rule of finding the solution (91).

Theorem 8. Suppose that $\mathbf{A}_{\mathscr{C}(\mathbf{A})}^{(-1)}$ exists. Then,

$$
x_{i}=\frac{\sum_{\beta \in J_{s, n}\{i\}}\left|\left(\mathbf{A}^{2}\right)_{. i}(\tilde{\mathbf{b}})\right|_{\beta}^{\beta}}{\sum_{\beta \in J_{s, n}}\left|\mathbf{A}^{2}\right|_{\beta}^{\beta} \sum_{\alpha \in I_{s, n}}\left|\mathbf{A} \mathbf{A}^{*}\right|_{\alpha}^{\alpha}},
$$

where $\widetilde{\mathbf{b}}:=\mathbf{U}_{2} \mathbf{b}, \mathbf{U}_{2}=\left(u_{i j}^{(2)}\right)$ is such that

$$
\mathbf{u}_{i j}^{(2)}=\sum_{\alpha \in I_{s, n}\{j\}}\left|\left(\mathbf{A A}^{*}\right)_{j .}\left(\widetilde{\mathbf{a}}_{i .}\right)\right|_{\alpha}^{\alpha},
$$

and $\widetilde{a}_{i}$ are the ith row of $\widetilde{\mathbf{A}}:=\mathbf{A}^{2} \mathbf{A}^{*}$.

Proof. Suppose $\mathbf{b}=\left[b_{1}, \ldots, b_{n}\right]^{T}$ and $\mathbf{x}=\left[x_{1}, \ldots, x_{n}\right]^{T}$. Since the Bott-Duffin inverse $\mathbf{A}_{\mathscr{C}(\mathbf{A})}^{(-1)}=\left(a_{i j}^{(-1)}\right)$ coincides with the right core inverse $\mathbf{A}^{\oplus}$, then its determinantal representation can be obtained by Theorem 2 . We use the determinantal representation (22). So, for all $i=1, \ldots, n$, we have

$$
x_{i}=\sum_{k} a_{i k}^{(-1)} b_{k}=\sum_{k} a_{i k}^{\oplus} b_{k}=\frac{\sum_{k} \sum_{\beta \in I_{s, n}\{i\}}\left|\left(\mathbf{A}^{2}\right)_{. i}\left(\mathbf{u}_{. k}^{(2)}\right)\right|_{\beta}^{\beta} b_{k}}{\sum_{\beta \in J_{s, n}}\left|\mathbf{A}^{2}\right|_{\beta}^{\beta} \sum_{\alpha \in I_{s, n}}\left|\mathbf{A A}^{*}\right|_{\alpha}^{\alpha}} .
$$

Construct the matrix $\mathbf{U}_{2}$ with columns determined by (24) and denote $\widetilde{\mathbf{b}}:=\mathbf{U}_{2} \mathbf{b}$. Then, from using this denotation in (95), it follows (93).

It is evident that the solution $\mathbf{y}=\left[y_{1}, \ldots, y_{n}\right]^{T}$ from (92) by the components can be expressed as

$$
y_{i}=b_{i}-\tilde{x}_{i}
$$

where $\tilde{x}_{i}$ is the $i$ th component of the column vector $\widetilde{\mathbf{x}}=\mathbf{A x}$.

\section{An Example}

Consider the matrix

$$
\mathbf{A}=\left[\begin{array}{ccc}
2 & 0 & 0 \\
-\mathbf{i} & \mathbf{i} & \mathbf{i} \\
-\mathbf{i} & -\mathbf{i} & -\mathbf{i}
\end{array}\right]
$$

Since

$$
\begin{aligned}
\mathbf{A A}^{*} & =\left[\begin{array}{ccc}
4 & 2 \mathbf{i} & 2 \mathbf{i} \\
-2 \mathbf{i} & 3 & -1 \\
-2 \mathbf{i} & -1 & 3
\end{array}\right], \\
\mathbf{A}^{2} & =\left[\begin{array}{ccc}
4 & 0 & 0 \\
2-2 \mathbf{i} & 0 & 0 \\
-2-2 \mathbf{i} & 0 & 0
\end{array}\right], \\
\mathbf{A}^{3} & =\left[\begin{array}{ccc}
8 & 0 & 0 \\
4-4 \mathbf{i} & 0 & 0 \\
-4-4 \mathbf{i} & 0 & 0
\end{array}\right],
\end{aligned}
$$

then $\operatorname{rk} \mathbf{A}=2$ and $\operatorname{rkA}^{2}=\operatorname{rkA}^{3}=1$, then $k=\operatorname{Ind} \mathbf{A}=2$ and $r_{1}=1$. So, we shall find $\mathbf{A}^{\oplus}$ and $\mathbf{A}_{\oplus}$ by (40) and (41), respectively. Since

$$
\widehat{\mathbf{A}}=\mathbf{A}^{2}\left(\mathbf{A}^{3}\right)^{*}=16\left[\begin{array}{ccc}
2 & 1+\mathbf{i} & -1+\mathbf{i} \\
1-\mathbf{i} & 1 & \mathbf{i} \\
-1-\mathbf{i} & \mathbf{i} & 1
\end{array}\right]
$$


then by (40)

$$
a_{11}^{\oplus, r}=\frac{\sum_{\alpha \in I_{1,3}\{1\}}\left|\left(\mathbf{A}^{3}\left(\mathbf{A}^{3}\right)^{*}\right)_{1 .}\left(\widehat{a}_{1 .}\right)\right|_{\alpha}^{\alpha}}{\sum_{\alpha \in I_{1,3}}\left|\mathbf{A}^{3}\left(\mathbf{A}^{3}\right)^{*}\right|_{\alpha}^{\alpha}}=\frac{1}{4} .
$$

By similarly continuing, we get

$$
\mathbf{A}^{\oplus}=\frac{1}{8}\left[\begin{array}{ccc}
2 & 1+\mathbf{i} & -1+\mathbf{i} \\
1-\mathbf{i} & 1 & \mathbf{i} \\
-1-\mathbf{i} & \mathbf{i} & 1
\end{array}\right] .
$$

By analogy, according to (41), we have

$$
\mathbf{A}_{\oplus}=\frac{1}{2}\left[\begin{array}{lll}
1 & 0 & 0 \\
0 & 0 & 0 \\
0 & 0 & 0
\end{array}\right] .
$$

The DMP inverse $\mathbf{A}^{d, \dagger}$ can be find by Theorem 5. Since

$$
\widetilde{A}=\mathbf{A}^{3} \mathbf{A}^{*}=4\left[\begin{array}{ccc}
4 & 2 \mathbf{i} & 2 \mathbf{i} \\
2-2 \mathbf{i} & 1+\mathbf{i} & 1+\mathbf{i} \\
-2-2 \mathbf{i} & 1-\mathbf{i} & 1-\mathbf{i}
\end{array}\right],
$$

and $\operatorname{rk}\left(\mathbf{A}^{3}\right)=1$, then

$$
\begin{aligned}
& \mathbf{u}_{1}^{(1)}=\widetilde{a}_{1,}, \\
& \mathbf{u}_{2}^{(1)}=\widetilde{a}_{2,}, \\
& \mathbf{u}_{3}^{(1)}=\widetilde{a}_{3,} .
\end{aligned}
$$

Furthermore, by (47),

$$
\begin{aligned}
a_{11}^{d, \dagger} & =\frac{\sum_{\alpha \in I_{2,3}\{1\}}\left|\left(\mathbf{A A}^{*}\right)_{1 .}\left(\mathbf{u}_{1 .}^{(1)}\right)\right|_{\alpha}^{\alpha}}{\sum_{\beta \in J_{1,3}}\left|\mathbf{A}^{3}\right|_{\beta}^{\beta} \sum_{\alpha \in I_{2,3}}\left|\mathbf{A A}^{*}\right|_{\alpha}^{\alpha}} \\
& =\frac{1}{192}\left(\operatorname{det}\left[\begin{array}{cc}
16 & 8 \mathbf{i} \\
-2 \mathbf{i} & 3
\end{array}\right]+\operatorname{det}\left[\begin{array}{cc}
16 & 8 \mathbf{i} \\
-2 & 3
\end{array}\right]\right)=\frac{1}{3} .
\end{aligned}
$$

By similarly continuing, we get

$$
\mathbf{A}^{d, \dagger}=\frac{1}{12}\left[\begin{array}{ccc}
4 & 2 \mathbf{i} & 2 \mathbf{i} \\
2-2 \mathbf{i} & 1+\mathbf{i} & 1+\mathbf{i} \\
-2-2 \mathbf{i} & 1-\mathbf{i} & 1-\mathbf{i}
\end{array}\right] .
$$

Similarly by Theorem 6 , we get

$$
\mathbf{A}^{\dagger, d}=\frac{1}{4}\left[\begin{array}{ccc}
2 & 0 & 0 \\
-\mathbf{i} & 0 & 0 \\
-\mathbf{i} & 0 & 0
\end{array}\right] .
$$

Finally, by Theorem, we find the CMP inverse $\mathbf{A}^{c, \dagger}=\left(a_{i j}^{c, \dagger}\right)$. Since $\operatorname{rk}^{3}=1$, then $\mathbf{G}=\widetilde{A}$ and

$$
\widetilde{G}=\mathbf{A}^{*} \mathbf{A} \widetilde{A}=16\left[\begin{array}{ccc}
6 & 3 \mathbf{i} & 3 \mathbf{i} \\
-2 \mathbf{i} & 1 & 1 \\
-2 \mathbf{i} & 1 & 1
\end{array}\right] .
$$

Furthermore, by (65),

$$
\begin{aligned}
w_{11}^{(2)} & =\sum_{\beta \in J_{2,3}\{1\}}\left|\left(\mathbf{A}^{*} \mathbf{A}\right)_{.1}\left(\widetilde{g}_{.1}\right)\right|_{\beta}^{\beta} \\
& =\left(\operatorname{det}\left[\begin{array}{cc}
6 & 0 \\
-2 \mathbf{i} & 2
\end{array}\right]+\operatorname{det}\left[\begin{array}{cc}
6 & 0 \\
-2 & 2
\end{array}\right]\right)=24 .
\end{aligned}
$$

By similar calculations, we get

$$
\begin{aligned}
& \mathbf{w}_{1 .}^{(2)}=[384,96 \mathbf{i}, 96 \mathbf{i}], \\
& \mathbf{w}_{2 .}^{(2)}=[-192 \mathbf{i}, 96,96], \\
& \mathbf{w}_{3 .}^{(2)}=[-192 \mathbf{i}, 96,06] .
\end{aligned}
$$

So, by (61), we get

$$
\begin{aligned}
a_{11}^{c, \dagger} & =\frac{\sum_{\alpha \in I_{2,3}\{1\}}\left|\left(\mathbf{A A}^{*}\right)_{1 .}\left(\mathbf{w}_{1 .}^{(2)}\right)\right|_{\alpha}^{\alpha}}{\left(\sum_{\alpha \in I_{2,3}}\left|\mathbf{A} \mathbf{A}^{*}\right|_{\alpha}^{\alpha}\right)^{2} \sum_{\beta \in J_{1,3}}\left|\mathbf{A}^{3}\right|_{\beta}^{\beta}} \\
& =\frac{1}{4608}\left(\operatorname{det}\left[\begin{array}{cc}
384 & 192 \mathbf{i} \\
-2 & 3
\end{array}\right]+\operatorname{det}\left[\begin{array}{cc}
384 & 192 \mathbf{i} \\
-2 & 3
\end{array}\right]\right)=\frac{1}{3} .
\end{aligned}
$$

By similarly continuing, we derive

$$
\mathbf{A}^{c, \dagger}=\frac{1}{12}\left[\begin{array}{ccc}
4 & 2 \mathbf{i} & 2 \mathbf{i} \\
-2 & 1 & 1 \\
-2 \mathbf{i} & 1 & 1
\end{array}\right] .
$$

\section{Conclusions}

In this chapter, we get the direct method to find of the core inverse and its generalizations that is based on their determinantal representations. New determinantal representations of the right and left core inverses, the right and left core-EP inverses, and the DMP, MPD, and CMP inverses are 
derived. Determinantal representation the Bott-Duffin inverse and its application to get Cramer's rule of the solution to the constrained linear equations are obtained.

\section{Data Availability}

No data were used to support this study.

\section{Conflicts of Interest}

The author declares that there are no conflicts of interest.

\section{References}

[1] O. M. Baksalary and G. Trenkler, "Core inverse of matrices," Linear and Multilinear Algebra, vol. 58, no. 6, pp. 681-697, 2010.

[2] S. Xu, J. Chen, and X. Zhang, "New characterizations for core inverses in rings with involution," Frontiers of Mathematics in China, vol. 12, no. 1, pp. 231-246, 2017.

[3] D. S. Rakić, N. Č. Dinčić, and D. S. Djordjević, "Group, Moore-Penrose, core and dual core inverse in rings with involution," Linear Algebra and Its Applications, vol. 463, pp. 115-133, 2014.

[4] K. M. Prasad and K. S. Mohana, "Core-EP inverse," Linear Multilinear Algebra, vol. 62, no. 6, pp. 792-802, 2014.

[5] O. M. Baksalary and G. Trenkler, "On a generalized core inverse," Applied Mathematics and Computation, vol. 236, pp. 450-457, 2014.

[6] S. B. Malik and N. Thome, "On a new generalized inverse for matrices of an arbitrary index," Applied Mathematics and Computation, vol. 226, pp. 575-580, 2014.

[7] M. Mehdipour and A. Salemi, "On a new generalized inverse of matrices," Linear and Multilinear Algebra, vol. 66, no. 5, pp. 1046-1053, 2018.

[8] J. Chen, H. Zhu, P. Patricio, and Y. Zhang, "Characterizations and representations of core and dual core inverses," Canadian Mathematical Bulletin, vol. 60, no. 2, pp. 269-282, 2017.

[9] Y. Gao and J. Chen, "Pseudo core inverses in rings with involution," Communications in Algebra, vol. 46, no. 1, pp. 38-50, 2018.

[10] A. Guterman, A. Herrero, and N. Thome, "New matrix partial order based on spectrally orthogonal matrix decomposition," Linear Multilinear Algebra, vol. 64, no. 3, pp. 362-374, 2016.

[11] D. E. Ferreyra, F. E. Levis, and N. Thome, "Maximal classes of matrices determining generalized inverses," Applied Mathematics and Computation, vol. 333, pp. 42-52, 2018.

[12] D. E. Ferreyra, F. E. Levis, and N. Thome, "Revisiting the core EP inverse and its extension to rectangular matrices," Quaestiones Mathematicae, vol. 41, no. 2, pp. 265-281, 2018.

[13] X. Liu and N. Cai, "High-order iterative methods for the DMP inverse," Journal of Mathematics, vol. 2018, Article ID 8175935, 6 pages, 2018.

[14] H. Ma and P. S. Stanimirović, "Characterizations, approximation and perturbations of the core-EP inverse," Applied Mathematics and Computation, vol. 359, pp. 404-417, 2019.

[15] J. Mielniczuk, "Note on the core matrix partial ordering," Discussiones Mathematicae Probability and Statistics, vol. 31, no. 1-2, pp. 71-75, 2011.

[16] D. Mosić, C. Deng, and H. Ma, "On a weighted core inverse in a ring with involution," Communications in Algebra, vol. 46, no. 6, pp. 2332-2345, 2018.
[17] K. M. Prasad and M. D. Raj, "Bordering method to compute core-EP inverse," Special Matrices, vol. 6, no. 1, pp. 193-200, 2018.

[18] H. Wang, "Core-EP decomposition and its applications," Linear Algebra and Its Applications, vol. 508, pp. 289-300, 2016.

[19] R. B. Bapat, K. P. S. Bhaskara Rao, and K. M. Prasad, "Generalized inverses over integral domains," Linear Algebra and Its Applications, vol. 140, pp. 181-196, 1990.

[20] K. P. S. Bhaskara Rao, "On generalized inverses of matrices over integral domains," Linear Algebra and Its Applications, vol. 49, pp. 179-189, 1983.

[21] I. I. Kyrchei, "Analogs of the adjoint matrix for generalized inverses and corresponding Cramer rules," Linear Multilinear Algebra, vol. 56, no. 4, pp. 453-469, 2008.

[22] I. Kyrchei, "Explicit formulas for determinantal representations of the Drazin inverse solutions of some matrix and differential matrix equations," Applied Mathematics and Computation, vol. 219, no. 14, pp. 7632-7644, 2013.

[23] I. Kyrchei, "Cramer's rule for generalized inverse solutions," in Advances in Linear Algebra Research, I. Kyrchei, Ed., pp. 79-132, Nova Science Publishers, New York, NY, USA, 2015.

[24] P. S. Stanimirović, "General determinantal representation of pseudoinverses of matrices," Matematički Vesnik, vol. 48, pp. 1-9, 1996.

[25] P. S. Stanimirović and D. S. Djordjevic, "Full-rank and determinantal representation of the Drazin inverse," Linear Algebra and Its Applications, vol. 311, no. 1-3, pp. 131-151, 2000.

[26] I. I. Kyrchei, "Determinantal representations of the MoorePenrose inverse over the quaternion skew field and corresponding Cramer's rules," Linear and Multilinear Algebra, vol. 59, no. 4, pp. 413-431, 2011.

[27] I. Kyrchei, "Determinantal representations of the Drazin inverse over the quaternion skew field with applications to some matrix equations," Applied Mathematics and Computation, vol. 238, pp. 193-207, 2014.

[28] I. I. Kyrchei, "Cramer's rule for quaternionic systems of linear equations," Journal of Mathematical Sciences, vol. 155, no. 6, pp. 839-858, 2008.

[29] I. Kyrchei, "The theory of the column and row determinants in a quaternion linear algebra," in Advances in Mathematics Research, A. R. Baswell, Ed., vol. 15, pp. 301-359, Nova Science Publishers, New York, NY, USA, 2012.

[30] I. Kyrchei, "Determinantal representations of the W-weighted Drazin inverse over the quaternion skew field," Applied Mathematics and Computation, vol. 264, pp. 453-465, 2015.

[31] I. Kyrchei, "Explicit determinantal representation formulas of W-weighted Drazin inverse solutions of some matrix equations over the quaternion skew field," Mathematical Problems in Engineering, vol. 2016, Article ID 8673809, 13 pages, 2016.

[32] I. Kyrchei, "Explicit determinantal representation formulas for the solution of the two-sided restricted quaternionic matrix equation," Journal of Applied Mathematics and Computing, vol. 58, no. 1-2, pp. 335-365, 2018.

[33] I. Kyrchei, "Determinantal representations of the Drazin and W-weighted Drazin inverses over the quaternion skew field with applications," in Quaternions: Theory and Applications, S. Griffin, Ed., pp. 201-275, Nova Science Publishers, New York, NY, USA, 2017.

[34] I. Kyrchei, "Weighted singular value decomposition and determinantal representations of the quaternion weighted 
Moore-Penrose inverse," Applied Mathematics and Computation, vol. 309, pp. 1-16, 2017.

[35] I. I. Kyrchei, "Determinantal representations of the quaternion weighted Moore-Penrose inverse and its applications," in Advances in Mathematics Research, R. B. Albert, Ed., vol. 23, pp. 35-96, Nova Science Publishers, New York, NY, USA, 2017.

[36] I. Kyrchei, "Cramer's Rules for Sylvester quaternion matrix equation and its special cases," Advances in Applied Clifford Algebra, vol. 28, no. 5, p. 90, 2018.

[37] I. I. Kyrchei, "Determinantal representations of general and (skew-)Hermitian solutions to the generalized Sylvester-type quaternion matrix equation," Abstract and Applied Analysis, vol. 2019, Article ID 5926832, 14 pages, 2019.

[38] I. I. Kyrchei, "Determinantal representations of solutions and Hermitian solutions to some system of two-sided quaternion matrix equations," Journal of Mathematics, vol. 2018, Article ID 6294672, 12 pages, 2018.

[39] G.-J. Song, Q.-W. Wang, and H.-X. Chang, "Cramer rule for the unique solution of restricted matrix equations over the quaternion skew field," Computers \& Mathematics with Applications, vol. 61, no. 6, pp. 1576-1589, 2011.

[40] G.-J. Song, "Determinantal representation of the generalized inverses over the quaternion skew field with applications," Applied Mathematics and Computation, vol. 219, no. 2, pp. 656-667, 2012.

[41] G. J. Song, "Bott-Duffin inverse over the quaternion skew field with applications," Journal of Applied Mathematics and Computing, vol. 41, no. 1-2, pp. 377-392, 2013.

[42] M. Zhou, J. Chen, T. Li, and D. Wang, "Three limit representations of the core-EP inverse," Filomat, vol. 32, no. 17, pp. 5887-5894, 2018.

[43] L. Wang, D. Mosić, and Y. Gao, "Right core inverse and the related generalized inverses," Communications in Algebra, vol. 47, no. 11, pp. 4749-4762, 2019.

[44] A. Ben-Israel and T. N. E. Greville, Generalized Inverses: Theory and Applications, Springer-Verlag, New York, NY, USA, 2nd edition, 2003.

[45] R. Bott and R. J. Duffin, "On the algebra of networks," Transactions of the American Mathematical Society, vol. 74, no. 1, pp. 99-109, 1953. 


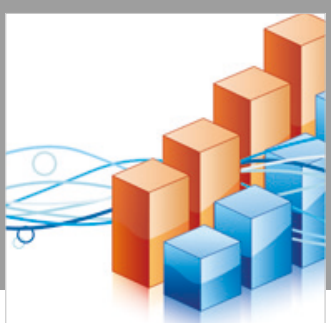

Advances in

Operations Research

\section{-n-m}
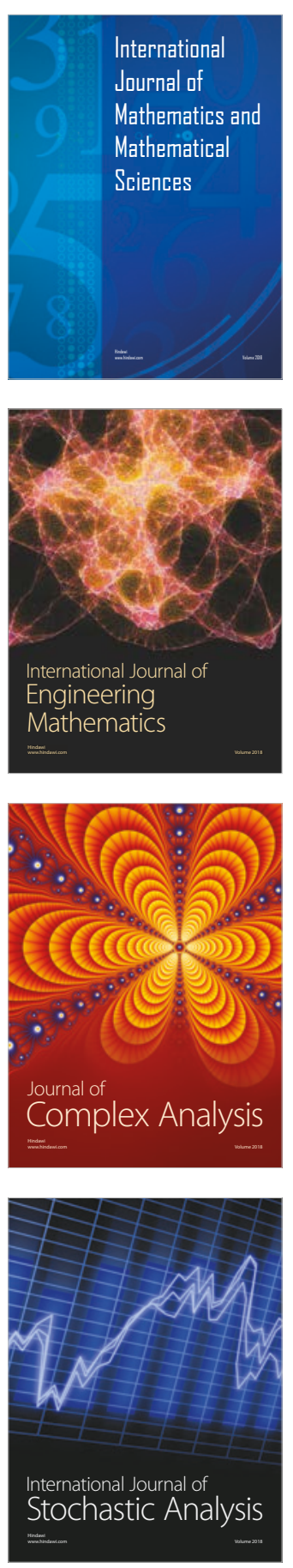
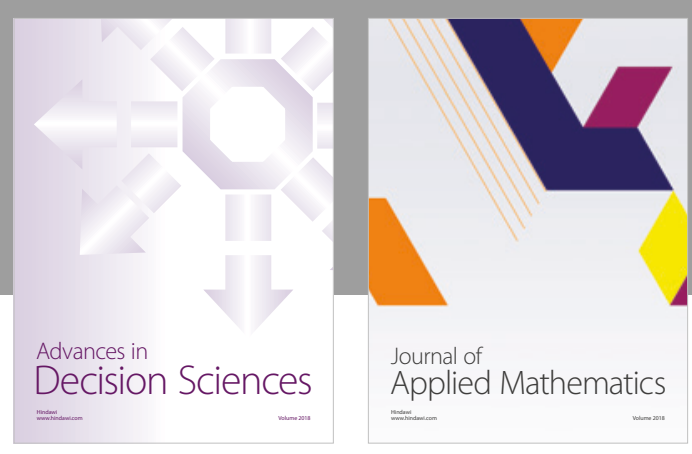

Journal of

Applied Mathematics
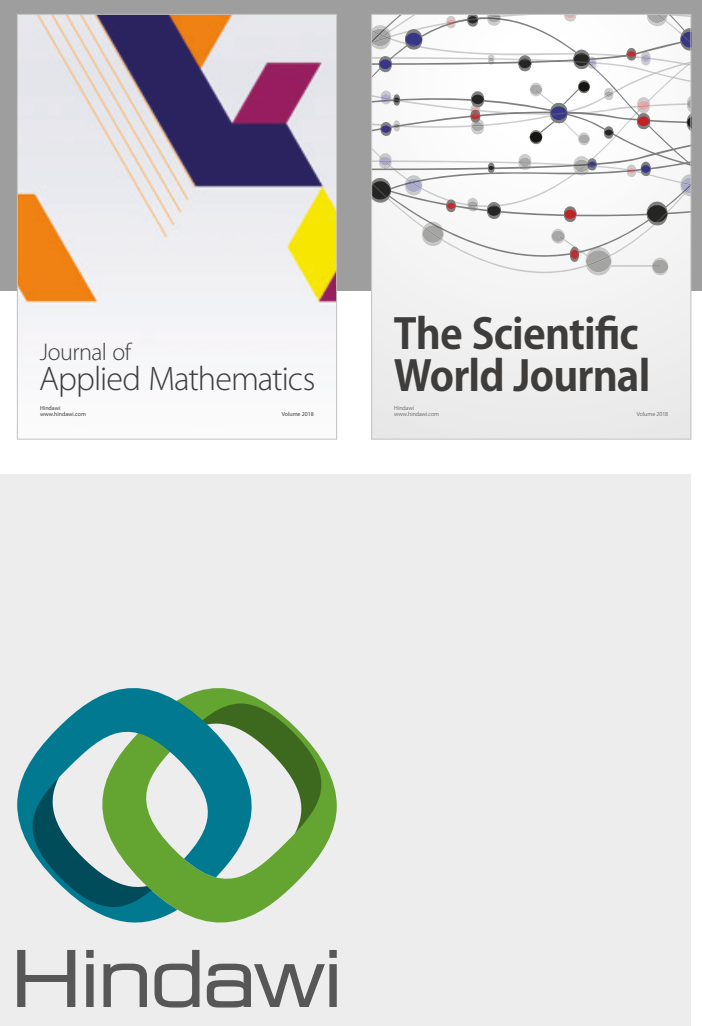

Submit your manuscripts at

www.hindawi.com

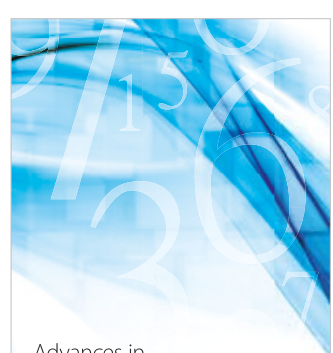

Advances in
Numerical Analysis
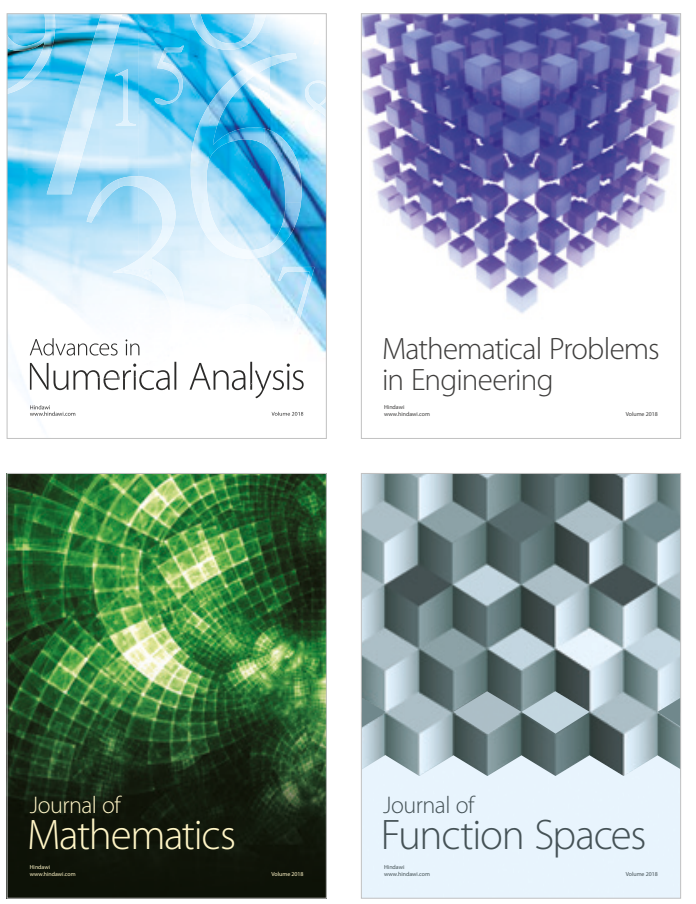

Mathematical Problems in Engineering

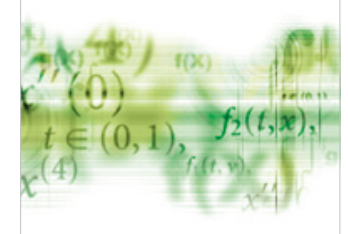

International Journal of

Differential Equations

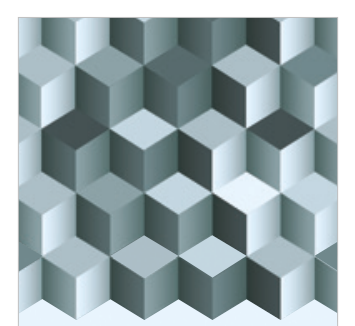

Journal of

Function Spaces

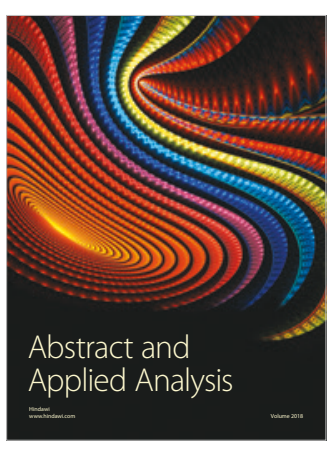

The Scientific

World Journal

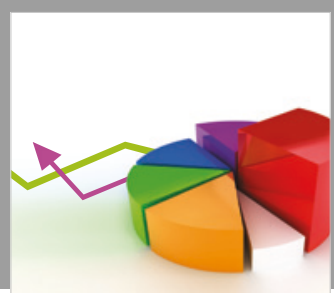

Journal of

Probability and Statistics
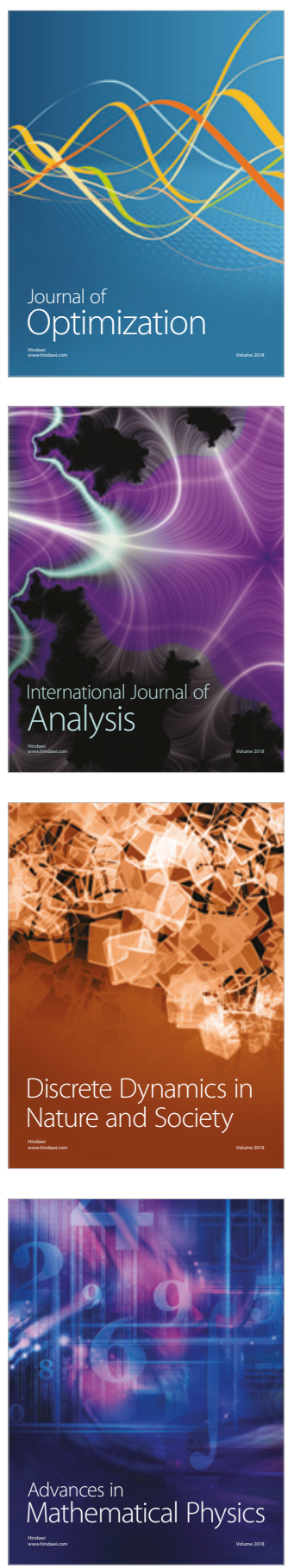